Proceedings of the Edinburgh Mathematical Society (2008) 51, 337-363 (C)

DOI:10.1017/S001309150600160X Printed in the United Kingdom

\title{
CONSTRUCTION OF ELLIPTIC DIFFUSIONS WITH REFLECTING BOUNDARY CONDITION AND AN APPLICATION TO CONTINUOUS $N$-PARTICLE SYSTEMS WITH SINGULAR INTERACTIONS
}

\author{
TORBEN FATTLER AND MARTIN GROTHAUS \\ Mathematics Department, University of Kaiserslautern, \\ PO Box 3049, 67653 Kaiserslautern, Germany \\ (fattler@mathematik.uni-kl.de; grothaus@mathematik.uni-kl.de)
}

(Received 15 December 2006)

Abstract We give a Dirichlet form approach for the construction and analysis of elliptic diffusions in $\bar{\Omega} \subset \mathbb{R}^{n}$ with reflecting boundary condition. The problem is formulated in an $L^{2}$-setting with respect to a reference measure $\mu$ on $\bar{\Omega}$ having an integrable, $\mathrm{d} x$-almost everywhere (a.e.) positive density $\varrho$ with respect to the Lebesgue measure. The symmetric Dirichlet forms $\left(\mathcal{E}^{\varrho}, a, D\left(\mathcal{E}^{\varrho}, a\right)\right)$ we consider are the closure of the symmetric bilinear forms

$$
\begin{gathered}
\mathcal{E}^{\varrho, a}(f, g)=\sum_{i, j=1}^{n} \int_{\Omega} \partial_{i} f a_{i j} \partial_{j} g \mathrm{~d} \mu, \quad f, g \in \mathcal{D}, \\
\mathcal{D}=\left\{f \in C(\bar{\Omega}) \mid f \in W_{\text {loc }}^{1,1}(\Omega), \mathcal{E}^{\varrho, a}(f, f)<\infty\right\},
\end{gathered}
$$

in $L^{2}(\bar{\Omega}, \mu)$, where $a$ is a symmetric, elliptic, $n \times n$-matrix-valued measurable function on $\bar{\Omega}$. Assuming that $\Omega$ is an open, relatively compact set with boundary $\partial \Omega$ of Lebesgue measure zero and that $\varrho$ satisfies the Hamza condition, we can show that $\left(\mathcal{E}^{\varrho, a}, D\left(\mathcal{E}^{\varrho, a}\right)\right)$ is a local, quasi-regular Dirichlet form. Hence, it has an associated self-adjoint generator $\left(L^{\varrho, a}, D\left(L^{\varrho}, a\right)\right)$ and diffusion process $\boldsymbol{M}^{\varrho, a}$ (i.e. an associated strong Markov process with continuous sample paths). Furthermore, since $1 \in D\left(\mathcal{E}^{\varrho, a}\right)$ (due to the Neumann boundary condition) and $\mathcal{E}^{\varrho, a}(1,1)=0$, we obtain a conservative process $\mathbf{M}^{\varrho}, a$ (i.e. $\boldsymbol{M}^{\varrho, a}$ has infinite lifetime). Additionally, assuming that $\sqrt{\varrho} \in W^{1,2}(\Omega) \cap C(\bar{\Omega})$ or that $\varrho$ is bounded, $\Omega$ is convex and $\{\varrho=0\}$ has codimension at least 2 , we can show that the set $\{\varrho=0\}$ has $\mathcal{E}^{\varrho, a}$-capacity zero. Therefore, in this case we can even construct an associated conservative diffusion process in $\{\varrho>0\}$. This is essential for our application to continuous $N$-particle systems with singular interactions. Note that for the construction of the self-adjoint generator $\left(L^{\varrho, a}, D\left(L^{\varrho, a}\right)\right)$ and the Markov process $M^{\varrho, a}$ we do not need to assume any differentiability condition on $\varrho$ and $a$. We obtain the following explicit representation of the generator for $\sqrt{\varrho} \in W^{1,2}(\Omega)$ and $a \in W^{1, \infty}(\Omega)$ :

$$
L^{\varrho, a}=\sum_{i, j=1}^{n} \partial_{i}\left(a_{i j} \partial_{j}\right)+\partial_{i}(\log \varrho) a_{i j} \partial_{j} .
$$

Note that the drift term can be singular, because we allow $\varrho$ to be zero on a set of Lebesgue measure zero. Our assumptions in this paper even allow a drift that is not integrable with respect to the Lebesgue measure.

Keywords: diffusion process; reflecting boundary condition; interacting continuous particle systems

2000 Mathematics subject classification: Primary 60H10; 60J60; 58J32; 60K35 


\section{Introduction}

The elliptic diffusions we construct in this paper are associated with symmetric Dirichlet forms $\left(\mathcal{E}^{\varrho, a}, D\left(\mathcal{E}^{\varrho, a}\right)\right)$ which are the closure of the symmetric bilinear forms

$$
\left.\begin{array}{c}
\mathcal{E}^{\varrho, a}(f, g)=\sum_{i, j=1}^{n} \int_{\Omega} \partial_{i} f a_{i j} \partial_{j} g \mathrm{~d} \mu, \quad f, g \in \mathcal{D}, \\
\mathcal{D}=\left\{f \in C(\bar{\Omega}) \mid f \in W_{\text {loc }}^{1,1}(\Omega), \mathcal{E}^{\varrho, a}(f, f)<\infty\right\}
\end{array}\right\}
$$

in $L^{2}(\bar{\Omega}, \mu)$. We assume that the measure $\mu$ on $\bar{\Omega} \subset \mathbb{R}^{n}$ has an integrable, dx-almost everywhere (a.e.) positive density $\varrho$ with respect to the Lebesgue measure. Furthermore, we assume $a$ to be a symmetric, elliptic, $n \times n$-matrix-valued measurable function on $\bar{\Omega}$. We assume that the set $\Omega$ is an open, relatively compact set with boundary $\partial \Omega$ of Lebesgue measure zero. $\bar{\Omega}$ denotes the closure of $\Omega$.

In the special case where $a$ is the identity matrix and $\varrho$ is a constant, the associated diffusion process is called reflected Brownian motion in $\bar{\Omega}$. It has been constructed and studied for $\Omega$ with Lipschitz boundary by Bass and Hsu $[\mathbf{5}, \mathbf{6}]$. (See also $[\mathbf{2 4}]$ for another approach.)

In the case where

$$
\sigma=\sqrt{a} \quad \text { and } \quad b=\left(\sum_{i=1}^{n} \partial_{i}(\log \varrho) a_{i j}\right)_{1 \leqslant j \leqslant n}
$$

are Lipschitz on $\bar{\Omega}$ and $\Omega$ is smooth, the process associated with $\left(\mathcal{E}^{\varrho, a}, D\left(\mathcal{E}^{\varrho, a}\right)\right)$ has been obtained as a solution to the corresponding stochastic differential equation by Lions and Sznitman [17].

Pardaux and Williams [21] investigated two methods for approximating the diffusion process associated with $\left(\mathcal{E}^{\varrho}, a, D\left(\mathcal{E}^{\varrho}, a\right)\right)$. One is a conventional penalty approximation by diffusions defined on all of $\mathbb{R}^{n}$. The other uses diffusions confined to $\bar{\Omega}$ by singular drifts that tend to infinity at the boundary of $\Omega$. Comparing our assumptions with those in $[\mathbf{2 1}]$, we assume only a stronger ellipticity of $a$. However, in [21], Pardaux and Williams assume, in addition to our conditions, that $a$ and $\varrho$ are locally Lipschitz. Furthermore, they assume that $\varrho>0$. We can allow $\varrho=0$ in $\Omega$ in a set of Lebesgue measure zero. This is essential for our application to continuous $N$-particle systems with singular interactions (see Theorem 5.4 and Remark 5.5). In the case where $\varrho$ is bounded above and below by positive constants, the diffusions we construct coincide with those obtained in $[\mathbf{2 1}]$ (see [21, Remark 3.10]).

Our approach is instead as in [2], where Albeverio et al. used Dirichlet form techniques (see $[\mathbf{1 0}, \mathbf{2 0}])$ to construct the diffusion corresponding to $\left(\mathcal{E}^{\varrho, a}, D\left(\mathcal{E}^{\varrho, a}\right)\right)$ in the case when $\Omega=\mathbb{R}^{n}$ and $a$ is the identity matrix. Our assumptions on $\varrho$ for constructing the diffusion process corresponding to $\left(\mathcal{E}^{\varrho, a}, D\left(\mathcal{E}^{\varrho, a}\right)\right)$ are still more general than those in $[\mathbf{2}]$ and are instead as used in [11], where Fukushima also considered the case $\Omega=\mathbb{R}^{n}$. In the case that we consider, with a compact $\bar{\Omega}$, however, we have to deal with other difficulties caused by the boundary (see Remarks 2.7 and 2.17). 
Furthermore, Trutnau [23] developed a Dirichlet form approach for the construction and analysis of reflected diffusions at the same time as we did. Among others, Trutnau [23] considers Dirichlet forms with the same assumptions on matrix $a$ and density $\varrho$ as we do. However, the diffusions studied in $[\mathbf{2 3}]$ correspond to Dirichlet forms obtained as the closure of $C^{\infty}(\bar{\Omega})$. For our application to continuous $N$-particle systems with singular interactions it is essential to have sufficiently many functions in $D\left(\mathcal{E}^{\varrho, a}\right)$ (see, for example, the proofs of Theorem 4.5, Corollary 4.7 and Proposition 5.3). Hence, we need to choose the Dirichlet form given by the closure of the larger space $\mathcal{D} \supset C^{\infty}(\bar{\Omega})$. In [23], after constructing the associated diffusion process $\boldsymbol{M}^{\varrho, a}$ by Dirichlet form techniques, a Skorokhod decomposition of $\boldsymbol{M}^{\varrho, a}$ is given. This, in particular, describes $\boldsymbol{M}^{\varrho, a}$ as a process with reflecting boundary condition. In the case where $\varrho$ is bounded above and below by positive constants, the diffusions we construct also coincide with those obtained in $[\mathbf{2 3}]$.

There are further articles on reflected diffusions (see, for example, $[\mathbf{7}, \mathbf{9}, \mathbf{1 2}]$ ) with results complementary to ours.

Our paper is organized as follows. In $\S 2$ we analyse the symmetric bilinear form (1.1). Assuming the Hamza condition (see Condition 2.2), we can show in Proposition 2.6 that $\left(\mathcal{E}^{\varrho, a}, \mathcal{D}\right)$ is closable. Hence, its closure $\left(\mathcal{E}^{\varrho, a}, D\left(\mathcal{E}^{\varrho, a}\right)\right)$ has an associated self-adjoint generator $\left(L^{\varrho, a}, D\left(L^{\varrho, a}\right)\right)$; see Remark 2.8. Furthermore, we can prove that $\left(\mathcal{E}^{\varrho, a}, D\left(\mathcal{E}^{\varrho, a}\right)\right)$ is a conservative, local, quasi-regular Dirichlet form (see Remark 2.7 (iv) and Propositions $2.11,2.16$ and 2.19). In order to simultaneously have closability, sufficient functions in $D\left(\mathcal{E}^{\varrho, a}\right)$ for our application to continuous $N$-particle systems and quasi-regularity, a proper choice of $\Omega$ and $\mathcal{D}$ is crucial (see Remarks 2.7 and 2.17). The main result of $\S 2$ is presented in Theorem 2.21, where we prove that $\left(\mathcal{E}^{\varrho, a}, D\left(\mathcal{E}^{\varrho, a}\right)\right)$ has an associated conservative diffusion process $\boldsymbol{M}^{o, a}$ taking values in $\bar{\Omega}$, i.e. an associated strong Markov process with continuous sample paths and infinite lifetime. Here, quasi-regularity gives the existence of the process $\boldsymbol{M}^{\varrho, a}$. Locality (see Proposition 2.19) implies that $\boldsymbol{M}^{\varrho, a}$ has continuous sample paths. The fact that $\boldsymbol{M}^{\varrho, a}$ is conservative (i.e. has an infinite lifetime) follows from $1 \in D\left(\mathcal{E}^{\varrho, a}\right)$ and $\mathcal{E}^{\varrho, a}(1,1)=0$. Furthermore, in Theorem 2.21 we prove that $\boldsymbol{M}^{\varrho, a}$ is the unique diffusion process having $\mu$ as symmetrizing measure and which solves the martingale problem for $\left(L^{\varrho, a}, D\left(L^{\varrho, a}\right)\right)$.

Since $M^{\varrho, a}$ solves the martingale problem for $\left(L^{\varrho, a}, D\left(L^{\varrho, a}\right)\right)$, it can be considered as the solution of a stochastic differential equation. Our existence result in Theorem 2.21, however, is so general that we do not even have an explicit formula for its generator, $\left(L^{\varrho, a}, D\left(L^{\varrho, a}\right)\right)$. Under the additional condition $\sqrt{\varrho} \in W^{1,2}(\Omega), a \in W^{1, \infty}(\Omega)$ and with $\Omega$ having Lipschitz boundary, in Theorem 3.2 we prove that

$$
\mathcal{D}_{\mathrm{N}}:=\left\{f \in W^{2, \infty}(\Omega) \mid \partial_{a \nu} f(x)=0 \text { for all } x \in \partial \Omega\right\} \subset D\left(L^{\varrho, a}\right),
$$

where $\nu$ denotes the outer normal with respect to $\partial \Omega$ and $a \nu$ is the linear transformation of $\nu$ under $a$. Furthermore, for all $f \in \mathcal{D}_{\mathrm{N}}$, we derive the representation

$$
L^{\varrho, a} f=\sum_{i, j=1}^{n} \partial_{i}\left(a_{i j} \partial_{j}\right) f+\partial_{i}(\ln \varrho) a_{i j} \partial_{j} f .
$$


Note that elements from $\mathcal{D}_{\mathrm{N}}$ have the Neumann boundary condition. We assume that $\Omega$ has Lipschitz boundary, so that the representation given in (1.3) holds for a larger class of functions from $D\left(L^{\varrho, a}\right)$. For functions with compact support in $\Omega$, we obtain the representation in (1.3) without assuming that $\Omega$ has Lipschitz boundary (see Remark 3.4). Now, using Itô's formula, we find that the process $M^{\varrho, a}$ solves the stochastic differential equation

$$
\mathrm{d} \boldsymbol{X}_{t}=b\left(\boldsymbol{X}_{t}\right) \mathrm{d} t+\sqrt{2 a}\left(\boldsymbol{X}_{t}\right) \mathrm{d} \boldsymbol{B}_{t} \text { inside } \Omega, \text { with reflecting boundary condition, }
$$

for $\mathcal{E}^{\varrho, a}$-quasi all initial conditions in $\boldsymbol{X}_{0} \in \bar{\Omega}$. Here, a solution is understood in the sense of the associated martingale problem and $\left(\boldsymbol{B}_{t}\right)_{t \geqslant 0}$ is a vector-valued Brownian motion. The function $b$ is defined as in (1.2).

In $\S 4$ we analyse $\left(\mathcal{E}^{\varrho}, a, D\left(\mathcal{E}^{\varrho}, a\right)\right)$ from the potential theoretical point of view. Assuming that $\sqrt{\varrho} \in W^{1,2}(\Omega) \cap C(\bar{\Omega})$ or that $\varrho$ is bounded, $\Omega$ is convex and $\{\varrho=0\}$ has at least codimension 2 , in Theorem 4.5 we can prove that the set $\{\varrho=0\}$ has $\mathcal{E}^{\varrho, a}$-capacity zero. Thus, we can restrict the Dirichlet form $\left(\mathcal{E}^{\varrho, a}, D\left(\mathcal{E}^{\varrho, a}\right)\right)$ to $\{\varrho>0\}$ as a conservative, local, quasi-regular Dirichlet form (see Corollary 4.7). This gives us an associated conservative diffusion process in $\{\varrho>0\}$ (see Corollary 4.8).

Finally, as an application we construct a solution to the $\mathrm{N}$-particle stochastic dynamics in $\Lambda \subset \mathbb{R}^{d}$. This dynamic takes values in the space of $N$-point configurations in $\Lambda$,

$$
\Gamma_{\Lambda}^{(N)}:=\{\gamma \subset \Lambda \mid \#(\gamma)=N\},
$$

and solves weakly the following $N$-system of stochastic differential equations:

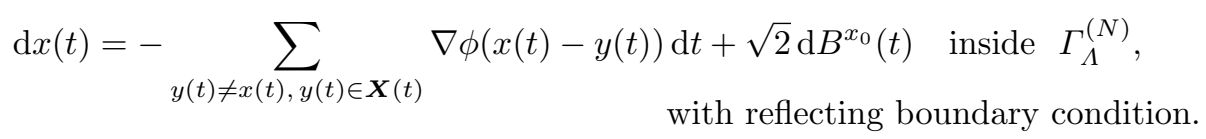

Here $x(t) \in \boldsymbol{X}(t) \in \Gamma_{\Lambda}^{(N)}$ and $\left(B^{x_{0}}\right)_{x_{0} \in \gamma_{0}}$ are $N$ independent Brownian motions starting in $x_{0}$. We prove in Theorem 5.4 the existence of a weak solution to (1.5) for all initial conditions $\gamma_{0} \in \Gamma_{\Lambda}^{(N)}$ except for a set of capacity zero. Our assumptions on the interaction potential allow singular interactions. In the case when $d=1$ we assume the interaction potential $\phi$ to be either strongly repulsive (SRP) and bounded below (BB), or repulsive (RP) and weakly differentiable $\left(\mathrm{DL}^{2}\right)$. In the case when $d \geqslant 2$ we must assume the interaction potential $\phi$ to be either repulsive (RP) and bounded below (BB), or just bounded; see below for a precise definition of (SRP), (RP), (BB) and $\left(\mathrm{DL}^{2}\right)$. In our construction, we first consider the corresponding Dirichlet form $\left(\mathcal{E}_{\Lambda, N}, D\left(\mathcal{E}_{\Lambda, N}\right)\right)$ on $\Lambda^{N} \subset$ $\mathbb{R}^{n}, n=N d$. The measure $\mu$ in this case is the canonical Gibbs measure corresponding to $N$ interacting particles in $\Lambda$. Then due to (RP), or in the case of a bounded potential by capacity estimates provided in [22], we find that the set of diagonals $D g$ in $\Lambda^{N}$ has $\mathcal{E}_{\Lambda, N}$-capacity zero (see Remark 5.5 ). Hence, via the symmetry mapping

$$
\begin{gathered}
\operatorname{sym}_{\Lambda}^{(N)}: \Lambda^{N} \backslash D g \rightarrow \Gamma_{\Lambda}^{(N)}, \\
\operatorname{sym}_{\Lambda}^{(N)}\left(x_{1}, \ldots, x_{N}\right)=\left\{x_{1}, \ldots, x_{N}\right\},
\end{gathered}
$$

we can construct a solution to (1.5). 
The following list of main results summarizes the progress achieved in this paper.

(i) We construct conservative diffusion processes with reflecting boundary condition under very mild assumptions on the drift part and diffusion part (see Theorem 2.21).

(ii) We provide an explicit representation of the generator for functions with Neumann boundary condition (see Theorem 3.2); this representation enables us, via the martingale problem, to identify the processes we construct as weak solutions to the stochastic differential equation (1.4).

(iii) We show that the set on which the density $\varrho$ of the symmetrizing measure $\mu$ is zero has capacity zero (see Theorem 4.5). As a corollary, we can construct the associated process on $\{\varrho>0\}$ (see Corollary 4.8).

(iv) We construct the $N$-particle, finite volume stochastic dynamics with reflecting boundary condition for singular interactions (see Theorem 5.4).

We consider this paper as a basis for several other articles. For example, it provides the $N$-particle dynamics in a finite volume for singular interactions, which is essential for proving an $N / V$-limit for infinite particle, infinite volume stochastic dynamics in continuous particle systems (see $[\mathbf{1 6}]$ ). Furthermore, in $[\mathbf{8}, \mathbf{1 5}]$ we analyse strong Feller properties and determine the spectral gap of the generators of the diffusions that we construct here.

It might be possible to construct $N$-particle dynamics for singular interactions by first regularizing the potential, using existing theory on stochastic differential equations to construct the corresponding approximating process and then attempting to take a weak limit. But then the question of whether the weak limit solves the associated martingale problem is still open. This property is important for the considerations in [16] and follows directly from the Dirichlet form approach. Furthermore, for the considerations in [16] a Lyons-Zheng decomposition (see $[\mathbf{1 8}, \mathbf{1 9}]$ ) of the $N$-particle dynamics into a forward and backward martingale is needed. The existence of such a decomposition is only guaranteed for processes associated with Dirichlet forms.

The reflecting boundary conditions are needed to obtain a process with an infinite lifetime. Note that Dirichlet form techniques allow such general boundaries (i.e. more general than Lipschitz) that even the notion of a reflection might not be well defined. Hence, for such boundaries we have a reflection at the boundary in a generalized sense.

\section{Dirichlet forms}

We start with the symmetric bilinear form

$$
\mathcal{E}^{\varrho, a}(f, g)=\sum_{i, j=1}^{n} \int_{\Omega} \partial_{i} f a_{i j} \partial_{j} g \mathrm{~d} \mu
$$


on $L^{2}(\bar{\Omega}, \mu)$. Throughout the paper we assume $a$ to be a symmetric, $n \times n$-matrix-valued measurable function that is uniformly globally strictly elliptic on $\Omega$, i.e. there exists $\kappa>0$ such that

$$
\kappa^{-1} \sum_{i=1}^{n} \xi_{i}^{2} \leqslant \sum_{i, j=1}^{n} a_{i j}(x) \xi_{i} \xi_{j} \leqslant \kappa \sum_{i=1}^{n} \xi_{i}^{2} \quad \text { for all } \xi=\left(\xi_{1}, \ldots, \xi_{n}\right) \in \mathbb{R}^{n}, \mu \text {-a.e. } x \in \Omega .
$$

We assume $\Omega$ to be an open, relatively compact set with boundary $\partial \Omega$ of Lebesgue measure zero. We assume the measure $\mu$ to have an integrable, $\mathrm{d} x$-a.e. positive density with respect to the Lebesgue measure, i.e. $\mu=\varrho \mathrm{d} x$, where $\varrho>0 \mathrm{~d} x$-a.e. on $\bar{\Omega}$ and $\varrho \in L^{1}(\bar{\Omega}, \mathrm{d} x)$. As a domain of $\mathcal{E}^{\varrho, a}$ we consider

$$
\mathcal{D}=\left\{f \in C(\bar{\Omega}) \mid f \in W_{\text {loc }}^{1,1}(\Omega), \mathcal{E}^{\varrho, a}(f, f)<\infty\right\} .
$$

Here $W_{\text {loc }}^{1,1}(\Omega)$ denotes the Sobolev space of weakly differentiable, locally integrable functions on $\Omega$.

\subsection{Closability of the bilinear form $\left(\mathcal{E}^{\varrho, a}, \mathcal{D}\right)$}

We start by recalling some basic facts on bilinear forms. For a detailed study see, for example, $[\mathbf{1 0}, \mathbf{2 0}]$.

Definition 2.1. A bilinear form $(\mathcal{E}, D)$ on $L^{2}(\bar{\Omega}, \mu)$ is said to be

(i) closed if the space $D$ is dense in $L^{2}(\bar{\Omega}, \mu)$ and complete with respect to the inner product

$$
\mathcal{E}_{1}(f, g)=\mathcal{E}(f, g)+(f, g)_{L^{2}(\bar{\Omega}, \mu)},
$$

where

$$
(f, g)_{L^{2}(\bar{\Omega}, \mu)}=\int_{\bar{\Omega}} f(x) g(x) \mu(\mathrm{d} x)
$$

(ii) closable if the condition

$$
\begin{aligned}
\text { if } f_{k} \in D, \mathcal{E}\left(f_{k}-f_{l}, f_{k}-f_{l}\right) \rightarrow 0 & \text { as } k, l \rightarrow \infty \\
& \text { and }\left(f_{k}, f_{k}\right)_{L^{2}(\bar{\Omega}, \mu)} \rightarrow 0 \text { as } k \rightarrow \infty, \text { then } \mathcal{E}\left(f_{k}, f_{k}\right) \rightarrow 0 \text { as } k \rightarrow \infty
\end{aligned}
$$

is satisfied.

In our considerations, the natural question of the conditions under which the form $\left(\mathcal{E}^{e, a}, \mathcal{D}\right)$ is closable arises. A discussion of this problem can be found, for example, in [11]. To prove closability of such a form, we have to set some additional restrictions on the density, $\varrho$. We define

$$
R_{\varrho}(\Omega):=\left\{x \in \Omega \mid \int_{\{y \in \Omega|| x-y \mid \leqslant \varepsilon\}} \varrho^{-1}(y) \mathrm{d} y<\infty \text { for some } \varepsilon>0\right\} .
$$

\section{Condition 2.2 (Hamza condition).}

$$
\varrho=0 \mathrm{~d} x \text {-a.e. on } \Omega \backslash R_{\varrho}(\Omega) .
$$


Remark 2.3. $R_{\varrho}(\Omega)$ is open and $\varrho>0 \mathrm{~d} x$-a.e. on $R_{\varrho}(\Omega)$. Obviously, $R_{\varrho}(\Omega)$ is the largest open set in $\Omega$ such that $\varrho^{-1} \in L_{\text {loc }}^{1}\left(R_{\varrho}(\Omega), \mathrm{d} x\right)$ (see, for example, [20, Chapter 2]).

Remark 2.4. Note that, due to the assumption that $\varrho>0 \mathrm{~d} x$-a.e. on $\bar{\Omega}$ and Condition 2.2, we obtain that $\Omega \backslash R_{\varrho}(\Omega)$ is of Lebesgue measure zero.

The next lemma will give us an estimate which is essential for proving closability of $\left(\mathcal{E}^{\varrho, a}, \mathcal{D}\right)$. For a proof see [20, Chapter II, Lemma 2.2].

Lemma 2.5. Let Condition 2.2 be satisfied, let $\varphi \in C_{0}^{\infty}\left(R_{\varrho}(\Omega)\right)$ and let $f \in L^{2}(\bar{\Omega}, \mu)$. There then exists $C_{1}(\varphi)<\infty$ such that

$$
\left|\int_{R_{\varrho}(\Omega)} f \varphi \mathrm{d} x\right| \leqslant C_{1}(\varphi) \cdot\|f\|_{L^{2}(\bar{\Omega}, \mu)} .
$$

Proposition 2.6. Consider the measure $\mu=\varrho \mathrm{d} x$ with density function $\varrho$ and suppose that Condition 2.2 is satisfied. Then the symmetric bilinear form

$$
\mathcal{E}^{\varrho, a}(f, g)=\sum_{i, j=1}^{n} \int_{\Omega} \partial_{i} f a_{i j} \partial_{j} g \mathrm{~d} \mu
$$

with domain

$$
\mathcal{D}=\left\{f \in C(\bar{\Omega}) \mid f \in W_{\text {loc }}^{1,1}(\Omega), \mathcal{E}^{\varrho, a}(f, f)<\infty\right\}
$$

is closable on $L^{2}(\bar{\Omega}, \mu)$. We denote the closure by $\left(\mathcal{E}^{\varrho, a}, D\left(\mathcal{E}^{\varrho, a}\right)\right)$.

Proof. Because of the ellipticity of $a$, we can restrict ourselves to the case where $a$ equals the identity on $\mathbb{R}^{n}$. Throughout the paper we write $\mathcal{E}^{\varrho}, a=\mathcal{E}^{\varrho}$, if $a$ equals the identity matrix. Let $\left(f_{k}\right)_{k \in \mathbb{N}}$ be a Cauchy sequence in $\mathcal{D}$ with respect to $\mathcal{E}^{\varrho}$, i.e.

$$
\mathcal{E}^{\varrho}\left(f_{k}-f_{l}, f_{k}-f_{l}\right) \rightarrow 0 \quad \text { as } k, l \rightarrow \infty .
$$

Suppose, furthermore, that $f_{k} \rightarrow 0$ in $L^{2}(\bar{\Omega}, \mu)$, i.e.

$$
\left(f_{k}, f_{k}\right)_{L^{2}(\bar{\Omega}, \mu)} \rightarrow 0 \quad \text { as } k \rightarrow \infty .
$$

We must check whether $\mathcal{E}^{\varrho}\left(f_{k}, f_{k}\right) \rightarrow 0$ as $k \rightarrow \infty$ (see Definition 2.1 (ii)).

We know that, for fixed $i \in\{1, \ldots, n\},\left(\partial_{i} f_{k}\right)_{k \in \mathbb{N}}$ converges to some $h_{i}$ in $L^{2}(\bar{\Omega}, \mu)$, since $\left(\partial_{i} f_{k}\right)_{k \in \mathbb{N}}$ is a Cauchy sequence in $L^{2}(\bar{\Omega}, \mu)$ and $\left(L^{2}(\bar{\Omega}, \mu),\|\cdot\|_{L^{2}(\bar{\Omega}, \mu)}\right)$ is complete. Now we use Lemma 2.5 for $\varphi \in C_{0}^{\infty}\left(R_{\varrho}(\Omega)\right)$ to obtain

$$
\begin{aligned}
\left|\int_{R_{\varrho}(\Omega)} h_{i} \varphi \mathrm{d} x-\int_{R_{\varrho}(\Omega)} \partial_{i} f_{k} \varphi \mathrm{d} x\right| & \leqslant \int_{R_{\varrho}(\Omega)}\left|\partial_{i} f_{k}-h_{i}\right||\varphi| \mathrm{d} x \\
& \leqslant C_{1}(\varphi)\left\|\partial_{i} f_{k}-h_{i}\right\|_{L^{2}(\bar{\Omega}, \mu)} \rightarrow 0 \quad \text { as } k \rightarrow \infty .
\end{aligned}
$$

Thus,

$$
\int_{R_{\varrho}(\Omega)} h_{i} \varphi \mathrm{d} x=\lim _{k \rightarrow \infty} \int_{R_{\varrho}(\Omega)} \partial_{i} f_{k} \varphi \mathrm{d} x
$$


This, together with an integration by parts, Hölder's inequality and the fact that

$$
\left(f_{k}, f_{k}\right)_{L^{2}(\bar{\Omega}, \mu)} \rightarrow 0 \quad \text { as } k \rightarrow \infty
$$

implies that

$$
\int_{R_{\varrho}(\Omega)} h_{i} \varphi \mathrm{d} x=\lim _{k \rightarrow \infty} \int_{R_{\varrho}(\Omega)} \partial_{i} f_{k} \varphi \mathrm{d} x=-\lim _{k \rightarrow \infty} \int_{R_{\varrho}(\Omega)} f_{k} \partial_{i} \varphi \mathrm{d} x=0 .
$$

Hence, $h_{i}$ is the zero element in the space $L^{2}\left(R_{\varrho}(\varrho), \mu\right)$. Thus, $h_{i}$ is the zero element in the space $L^{2}(\bar{\Omega}, \mu)$, since $\partial \Omega$ has Lebesgue measure zero and $\varrho=0$ on $\Omega \backslash R^{\varrho}(\Omega)$ by Condition 2.2. Thus, we have proven that $\mathcal{E}^{\varrho}\left(f_{k}, f_{k}\right) \rightarrow 0$ as $k \rightarrow \infty$.

\section{Remark 2.7.}

(i) Note that the proof of Proposition 2.6 is based on the fact that $\partial \Omega$ has Lebesgue measure zero.

(ii) From the proof of Proposition 2.6 we can easily conclude that $\mathcal{E}^{\varrho, a}$ with the larger domain

$$
\widetilde{D\left(\mathcal{E}^{\varrho, a}\right)}:=\left\{f \in L^{2}(\bar{\Omega}, \mu) \mid f \in W_{\mathrm{loc}}^{1,1}\left(R_{\varrho}(\Omega)\right), \mathcal{E}^{\varrho, a}(f, f)<\infty\right\}
$$

is closed. In general, however, it is not clear whether $\mathcal{D}=C(\bar{\Omega}) \cap \widetilde{\left.D_{(\mathcal{E}}{ }^{\varrho, a}\right)}$ is dense in $\widehat{D\left(\mathcal{E}^{\varrho, a}\right)}$ with respect to $\sqrt{\mathcal{E}_{1}^{\varrho, a}}$. This property is needed to show quasi-regularity (see $\S 2.3$ ), which is essential for our construction of the associated Markov process in Theorem 2.21.

(iii) On the other hand, for our application to continuous $N$-particle systems, sufficiently many functions in the domain of $\mathcal{E}^{\varrho, a}$ are needed. For example, choosing $\mathcal{D}=C^{1}(\bar{\Omega})$, it is not clear whether the corresponding closure would have sufficiently many functions for proving that the set $\{\varrho=0\}$ has capacity zero; see the proof of Theorem 4.5. This theorem in fact is essential for our application to continuous $N$-particle systems (see Remark 5.5).

(iv) Since $1 \in \mathcal{D}$ and $\mathcal{E}^{\varrho, a}(1,1)=0$, the bilinear form $\left(\mathcal{E}^{\varrho, a}, D\left(\mathcal{E}^{\varrho, a}\right)\right)$ is conservative. In the case where $\left(\mathcal{E}^{\varrho, a}, D\left(\mathcal{E}^{\varrho, a}\right)\right)$ has an associated diffusion process $M^{\varrho, a}$ (see Theorem 2.21), this implies that $\boldsymbol{M}^{\varrho, a}$ has infinite lifetime.

\section{Notation.}

Recall that $\Omega \backslash R_{\varrho}(\Omega)$ has Lebesgue measure zero. Thus, after the considerations above we set $\nabla f:=\left(\partial_{1} f, \ldots, \partial_{n} f\right):=\left(h_{1}, \ldots, h_{n}\right)$ for all $f \in D\left(\mathcal{E}^{\varrho, a}\right)$.

Remark 2.8. By the Friedrichs representation theorem (see, for example, $[3$, Theorem 4]) we obtain the existence of the self-adjoint generator $\left(L^{\varrho, a}, D\left(L^{\varrho, a}\right)\right)$ corresponding to $\left(\mathcal{E}^{\varrho, a}, D\left(\mathcal{E}^{\varrho, a}\right)\right)$, i.e. $D\left(L^{\varrho, a}\right) \subset D\left(\mathcal{E}^{\varrho, a}\right)$ and

$$
\mathcal{E}^{\varrho, a}(f, g)=-\int_{\Omega} L^{\varrho, a} f g \mathrm{~d} \mu \quad \text { for all } f \in D\left(L^{\varrho, a}\right), g \in D\left(\mathcal{E}^{\varrho, a}\right) .
$$


Of course, $\left(L^{\varrho, a}, D\left(L^{\varrho, a}\right)\right)$ generates a strongly continuous contraction semi-group

$$
\left(T_{t}^{\varrho, a}\right)_{t \geqslant 0}:=\left(\exp \left(t L^{\varrho, a}\right)\right)_{t \geqslant 0}
$$

(see, for example, $[\mathbf{1 1}, \mathbf{2 0}]$ ).

\subsection{Markov property of $\left(\mathcal{E}^{\varrho}, \mathcal{D}\left(\mathcal{E}^{\varrho}\right)\right)$}

Definition 2.9. A symmetric closed bilinear form $(\mathcal{E}, D(\mathcal{E}))$ on $L^{2}(\bar{\Omega}, \mu)$ is called Markovian if one has

$$
f \in \mathcal{D}(\mathcal{E}) \text { implies } f^{+} \wedge 1 \in \mathcal{D}(\mathcal{E}) \text { and } \mathcal{E}\left(f^{+} \wedge 1, f^{+} \wedge 1\right) \leqslant \mathcal{E}(f, f) \text {, }
$$

where $f^{+}:=\max \{0, f\}$ and $f \wedge 1:=\min \{1, f\}$.

Remark 2.10. One can easily show that, for each $\varepsilon>0$, there exists a real function $\varphi_{\varepsilon}(t), t \in \mathbb{R}$, such that

$$
\begin{aligned}
\varphi_{\varepsilon}(t) & =t & & \text { for all } t \in[0,1] \\
-\varepsilon \leqslant \varphi_{\varepsilon}(t) & \leqslant 1+\varepsilon & & \text { for all } t \in \mathbb{R} \\
0 \leqslant \varphi_{\varepsilon}(s)-\varphi_{\varepsilon}(t) & \leqslant s-t, & & t<s .
\end{aligned}
$$

Then it is sufficient to check that

$$
f \in D(\mathcal{E}) \text { implies } \varphi_{\varepsilon}(f) \in D(\mathcal{E}) \text { and } \mathcal{E}\left(\varphi_{\varepsilon}(f), \varphi_{\varepsilon}(f)\right) \leqslant \mathcal{E}(f, f),
$$

to obtain that $(\mathcal{E}, D(\mathcal{E}))$ is Markovian (see, for example, [20, Chapter $1, \S 4]$ ).

Proposition 2.11. Suppose that Condition 2.2 is satisfied. Then $\left(\mathcal{E}^{\varrho, a}, D\left(\mathcal{E}^{\varrho, a}\right)\right)$ is Markovian. A Markovian form is also called a Dirichlet form.

Before we can prove the above proposition we need the following result from the theory of Sobolev spaces. For a proof we refer the reader to [14, Lemma 7.5].

Lemma 2.12. Let $f \in C^{1}(\mathbb{R}), f^{\prime} \in L^{\infty}(\mathbb{R})$ and $u \in W_{\mathrm{loc}}^{1,1}(\Omega)$. Then $f(u) \in W_{\mathrm{loc}}^{1,1}(\Omega)$ and

$$
\partial_{i}(f(u))=f^{\prime}(u) \partial_{i} u
$$

Proof of Proposition 2.11. As before, by the ellipticity of $a$ it is sufficient to consider the case where $a$ equals the identity matrix. Let $\varphi_{\varepsilon}$ be as in Remark 2.10 and let us take $f \in D\left(\mathcal{E}^{\varrho}\right)$. At first we consider $\varphi_{\varepsilon}(f)$ as a function in $L^{2}(\bar{\Omega}, \mu)$. Then we take $\left(f_{k}\right)_{k \in \mathbb{N}} \subset \mathcal{D}$ such that $f_{k} \rightarrow f$ in $\left(D\left(\mathcal{E}^{\varrho}\right), \sqrt{\mathcal{E}_{1}^{\varrho}}\right)$ and additionally $f_{k} \rightarrow f \mu$-a.e. as $k \rightarrow \infty$. Obviously, $\varphi_{\varepsilon}\left(f_{k}\right) \in C(\bar{\Omega})$ for all $k \in \mathbb{N}$. Since $\varphi_{\varepsilon} \in C^{1}(\mathbb{R}), \varphi_{\varepsilon}^{\prime} \in L^{\infty}(\mathbb{R})$ and $f_{k} \in W_{\text {loc }}^{1,1}(\Omega)$, we have $\varphi_{\varepsilon}\left(f_{k}\right) \in W_{\text {loc }}^{1,1}(\Omega)$ and $\partial_{i}\left(\varphi_{\varepsilon}\left(f_{k}\right)\right)=\varphi_{\varepsilon}^{\prime}\left(f_{k}\right) \partial_{i} f_{k}$ for all $k \in \mathbb{N}$, by Lemma 2.12. Furthermore, we have

$$
\left\|\partial_{i}\left(\varphi_{\varepsilon}\left(f_{k}\right)\right)\right\|_{L^{2}(\bar{\Omega}, \mu)}=\left\|\varphi_{\varepsilon}^{\prime}\left(f_{k}\right) \partial_{i} f_{k}\right\|_{L^{2}(\bar{\Omega}, \mu)} \leqslant\left\|\partial_{i} f_{k}\right\|_{L^{2}(\bar{\Omega}, \mu)}<\infty
$$


by the properties of $\varphi_{\varepsilon}$, and therefore $\varphi_{\varepsilon}\left(f_{k}\right) \in D\left(\mathcal{E}^{\varrho}\right)$ for all $k \in \mathbb{N}$. Clearly, $\varphi_{\varepsilon}\left(f_{k}\right) \rightarrow$ $\varphi_{\varepsilon}(f)$ in $L^{2}(\bar{\Omega}, \mu)$ as $k \rightarrow \infty$, since

$$
\left\|\varphi_{\varepsilon}\left(f_{k}\right)-\varphi_{\varepsilon}(f)\right\|_{L^{2}(\bar{\Omega}, \mu)} \leqslant\left\|f_{k}-f\right\|_{L^{2}(\bar{\Omega}, \mu)},
$$

again by the properties of $\varphi_{\varepsilon}$. Next we show that $\left(\varphi_{\varepsilon}\left(f_{k}\right)\right)_{k \in \mathbb{N}}$ is a Cauchy sequence in $\left(D\left(\mathcal{E}^{\varrho}\right), \sqrt{\mathcal{E}_{1}^{\varrho}}\right)$. Therefore, we consider

$$
\begin{aligned}
\mathcal{E}_{1}^{\varrho}\left(\varphi_{\varepsilon}\left(f_{k}\right)-\varphi_{\varepsilon}\left(f_{l}\right), \varphi_{\varepsilon}\left(f_{k}\right)-\varphi_{\varepsilon}\left(f_{l}\right)\right)= & \mathcal{E}^{\varrho}\left(\varphi_{\varepsilon}\left(f_{k}\right)-\varphi_{\varepsilon}\left(f_{l}\right), \varphi_{\varepsilon}\left(f_{k}\right)-\varphi_{\varepsilon}\left(f_{l}\right)\right) \\
& +\left(\varphi_{\varepsilon}\left(f_{k}\right)-\varphi_{\varepsilon}\left(f_{l}\right), \varphi_{\varepsilon}\left(f_{k}\right)-\varphi_{\varepsilon}\left(f_{l}\right)\right)_{L^{2}(\bar{\Omega}, \mu)}
\end{aligned}
$$

Since $\varphi_{\varepsilon}\left(f_{k}\right) \rightarrow \varphi_{\varepsilon}(f)$ in $L^{2}(\bar{\Omega}, \mu)$ we have

$$
\left(\varphi_{\varepsilon}\left(f_{k}\right)-\varphi_{\varepsilon}\left(f_{l}\right), \varphi_{\varepsilon}\left(f_{k}\right)-\varphi_{\varepsilon}\left(f_{l}\right)\right)_{L^{2}(\bar{\Omega}, \mu)} \rightarrow 0 \quad \text { as } k, l \rightarrow \infty .
$$

Thus, it remains to consider

$$
\begin{aligned}
\sum_{i=1}^{n} \int_{\Omega}\left(\partial_{i}\left(\varphi_{\varepsilon}\left(f_{k}\right)-\varphi_{\varepsilon}\left(f_{l}\right)\right)\right)^{2} \mathrm{~d} \mu & \\
= & \sum_{i=1}^{n} \int_{\Omega}\left(\varphi_{\varepsilon}^{\prime}\left(f_{k}\right) \partial_{i} f_{k}-\varphi_{\varepsilon}^{\prime}\left(f_{l}\right) \partial_{i} f_{l}\right)^{2} \mathrm{~d} \mu \quad \text { (by applying Lemma 2.12) } \\
= & \sum_{i=1}^{n} \int_{\Omega}\left(\varphi_{\varepsilon}^{\prime}\left(f_{k}\right)\left(\partial_{i} f_{k}-\partial_{i} f_{l}\right)+\left(\varphi_{\varepsilon}^{\prime}\left(f_{k}\right)-\varphi_{\varepsilon}^{\prime}\left(f_{l}\right)\right)\left(\partial_{i} f_{l}-\partial_{i} f+\partial_{i} f\right)\right)^{2} \mathrm{~d} \mu \\
\leqslant & 3\left(\left\|\varphi_{\varepsilon}^{\prime}\left(f_{k}\right)\right\|_{\text {sup }}^{2}\left\|\nabla f_{k}-\nabla f_{l}\right\|_{L^{2}(\bar{\Omega}, \mu)}^{2}+\left\|\left(\varphi_{\varepsilon}^{\prime}\left(f_{k}\right)-\varphi_{\varepsilon}^{\prime}\left(f_{l}\right)\right) \nabla f\right\|_{L^{2}(\bar{\Omega}, \mu)}^{2}\right. \\
\quad & \left.\quad\left\|\left(\varphi_{\varepsilon}^{\prime}\left(f_{k}\right)-\varphi_{\varepsilon}^{\prime}\left(f_{l}\right)\right)\right\|_{s u p}^{2}\left\|\nabla f_{l}-\nabla f\right\|_{L^{2}(\bar{\Omega}, \mu)}^{2}\right) \\
\leqslant & 3\left(\left\|\nabla f_{k}-\nabla f_{l}\right\|_{L^{2}(\bar{\Omega}, \mu)}^{2}+4\left\|\nabla f_{l}-\nabla f\right\|_{L^{2}(\bar{\Omega}, \mu)}^{2}\right. \\
\quad & \left.+2\left\|\left(\varphi_{\varepsilon}^{\prime}\left(f_{k}\right)-\varphi_{\varepsilon}^{\prime}(f)\right) \nabla f\right\|_{L^{2}(\bar{\Omega}, \mu)}^{2}+2\left\|\left(\varphi_{\varepsilon}^{\prime}(f)-\varphi_{\varepsilon}^{\prime}\left(f_{l}\right)\right) \nabla f\right\|_{L^{2}(\bar{\Omega}, \mu)}^{2}\right) .
\end{aligned}
$$

Since $\left|\left(\varphi_{\varepsilon}^{\prime}\left(f_{k}\right)-\varphi_{\varepsilon}^{\prime}(f)\right) \nabla f\right|$ and $\left|\left(\varphi_{\varepsilon}^{\prime}(f)-\varphi_{\varepsilon}^{\prime}\left(f_{l}\right)\right) \nabla f\right|$ are bounded by $g:=2|\nabla f| \epsilon$ $L^{2}(\bar{\Omega}, \mu)$ and $\varphi_{\varepsilon}^{\prime}\left(f_{k}\right) \rightarrow \varphi_{\varepsilon}^{\prime}(f) \mu$-a.e. as $k \rightarrow \infty$, by using Lebesgue's dominated convergence theorem we have that

$$
\left\|\left(\varphi_{\varepsilon}^{\prime}\left(f_{k}\right)-\varphi_{\varepsilon}^{\prime}(f)\right) \nabla f\right\|_{L^{2}(\bar{\Omega}, \mu)}+\left\|\left(\varphi_{\varepsilon}^{\prime}(f)-\varphi_{\varepsilon}^{\prime}\left(f_{l}\right)\right) \nabla f\right\|_{L^{2}(\bar{\Omega}, \mu)} \rightarrow 0 \quad \text { as } k, l \rightarrow \infty .
$$

Thus, (2.1) together with $f_{k} \rightarrow f$ in $\left(D\left(\mathcal{E}^{\varrho}\right), \sqrt{\mathcal{E}_{1}^{\varrho}}\right)$ implies that

$$
\mathcal{E}_{1}^{\varrho}\left(\varphi_{\varepsilon}\left(f_{k}\right)-\varphi_{\varepsilon}\left(f_{l}\right), \varphi_{\varepsilon}\left(f_{k}\right)-\varphi_{\varepsilon}\left(f_{l}\right)\right) \rightarrow 0 \quad \text { as } k, l \rightarrow \infty .
$$

Hence, $\left(\varphi_{\varepsilon}\left(f_{k}\right)\right)_{k \in \mathbb{N}}$ is a Cauchy sequence in $D\left(\mathcal{E}^{\varrho}\right)$ with respect to $\sqrt{\mathcal{E}_{1}^{\varrho}}$. Thus, it is convergent in $D\left(\mathcal{E}^{\varrho}\right)$ and

$$
\varphi_{\varepsilon}(f)=\lim _{k \rightarrow \infty} \varphi_{\varepsilon}\left(f_{k}\right) \in D\left(\mathcal{E}^{\varrho}\right)
$$


Furthermore,

$$
\begin{aligned}
\mathcal{E}^{\varrho}\left(\varphi_{\varepsilon}(f), \varphi_{\varepsilon}(f)\right) & =\lim _{k \rightarrow \infty} \mathcal{E}^{\varrho}\left(\varphi_{\varepsilon}\left(f_{k}\right), \varphi_{\varepsilon}\left(f_{k}\right)\right) \\
& =\lim _{k \rightarrow \infty} \sum_{i=1}^{n} \int_{\Omega}\left(\partial_{i} \varphi_{\varepsilon}\left(f_{k}\right)\right)^{2} \mathrm{~d} \mu \\
& =\lim _{k \rightarrow \infty} \sum_{i=1}^{n} \int_{\Omega} \underbrace{\left|\varphi_{\varepsilon}^{\prime}\left(f_{k}\right)\right|^{2}}_{\leqslant 1}\left(\partial_{i} f_{k}\right)^{2} \mathrm{~d} \mu \\
& \leqslant \lim _{k \rightarrow \infty} \sum_{i=1}^{n} \int_{\Omega}\left(\partial_{i} f_{k}\right)^{2} \mathrm{~d} \mu \\
& =\mathcal{E}^{\varrho}(f, f) .
\end{aligned}
$$

Thus, $\left(\mathcal{E}^{\varrho}, D\left(\mathcal{E}^{\varrho}\right)\right)$ is Markovian.

\subsection{Quasi-regularity of the Dirichlet form $\left(\mathcal{E}^{\varrho, a}, D\left(\mathcal{E}^{\varrho, a}\right)\right)$}

To get started with quasi-regularity, we have to introduce some notions from analytic potential theory of Dirichlet forms. A detailed discussion of the theory needed in this section can be found in $[\mathbf{2 0}$, Chapter III]. In this section $(\mathcal{E}, D(\mathcal{E}))$ denotes a Dirichlet form on $L^{2}(\bar{\Omega}, \mu)$.

\section{Definition 2.13.}

(i) An increasing sequence $\left(F_{k}\right)_{k \in \mathbb{N}}$ of closed subsets of $\bar{\Omega}$ is called an $\mathcal{E}$-nest if $\bigcup_{k \geqslant 1} D(\mathcal{E})_{F_{k}}$ is dense in $D(\mathcal{E})$ with respect to $\sqrt{\mathcal{E}_{1}}$, where

$$
D(\mathcal{E})_{F_{k}}:=\left\{u \in D(\mathcal{E}) \mid u=0 \mu \text {-a.e. on } \bar{\Omega} \backslash F_{k}\right\} .
$$

(ii) A subset $N \subset \Omega$ is called $\mathcal{E}$-exceptional if $N \subset \bigcap_{k \geqslant 1}\left(\bar{\Omega} \backslash F_{k}\right)$ for some $\mathcal{E}$-nest $\left(F_{k}\right)_{k \in \mathbb{N}}$. We say that a property of points in $\Omega$ holds $\mathcal{E}$-quasi-everywhere $(\mathcal{E}$-q.e.) if the property holds outside some $\mathcal{E}$-exceptional set.

Next we introduce the notion of quasi-continuity.

Definition 2.14. An $\mathcal{E}$-q.e. defined function $f$ on $\bar{\Omega}$ is called $\mathcal{E}$-quasi continuous if there exists an $\mathcal{E}$-nest $\left(F_{k}\right)_{k \in \mathbb{N}}$ such that

$$
f \in C\left(\left\{F_{k}\right\}\right):=\left\{f: A \rightarrow \mathbb{R}\left|\bigcup_{k \geqslant 1} F_{k} \subset A \subset \bar{\Omega}, f\right|_{F_{k}} \text { is continuous for every } k \in \mathbb{N}\right\} .
$$

We can now define quasi-regularity, as follows.

Definition 2.15. A Dirichlet form $(\mathcal{E}, D(\mathcal{E}))$ on $L^{2}(\bar{\Omega}, \mu)$ is called quasi-regular if there exists

(i) an $\mathcal{E}$-nest $\left(F_{k}\right)_{k \in \mathbb{N}}$ consisting of compact sets,

(ii) an $\sqrt{\mathcal{E}_{1}}$-dense subset of $D(\mathcal{E})$ whose elements have $\mathcal{E}$-quasi-continuous $\mu$-versions, 
(iii) a sequence of functions $u_{l} \in D(\mathcal{E}), l \in \mathbb{N}$, having $\mathcal{E}$-quasi-continuous $\mu$-versions $\tilde{u}_{l}$, $l \in \mathbb{N}$, and an $\mathcal{E}$-exceptional set $N \subset \bar{\Omega}$ such that $\left\{\tilde{u}_{l} \mid l \in \mathbb{N}\right\}$ separates the points of $\bar{\Omega} \backslash N$.

We can now state the main result of this section.

Proposition 2.16. Suppose that Condition 2.2 is satisfied. Then $\left(\mathcal{E}^{\varrho, a}, D\left(\mathcal{E}^{\varrho, a}\right)\right)$ is quasi-regular.

Proof. Let us check whether Definition 2.15 (i)-(iii) hold. Obviously, $\left(F_{k}\right)_{k \in \mathbb{N}}$, $F_{k}=\bar{\Omega}, k \in \mathbb{N}$, is an $\mathcal{E}^{\varrho, a}$-nest consisting of compact sets. Since $D\left(\mathcal{E}^{\varrho, a}\right)$ is the completion of $\mathcal{D}$ with respect to $\sqrt{\mathcal{E}_{1}^{\varrho, a}}$, we see that $\mathcal{D} \subset C(\bar{\Omega})$ is dense in $D\left(\mathcal{E}^{\varrho, a}\right)$ with respect to $\sqrt{\mathcal{E}_{1}^{\varrho, a}}$ and thus property (ii) is proved.

It remains to find a sequence of functions $\left\{u_{l} \in \mathcal{D}, l \in \mathbb{N}\right\}$ which separates points in $\bar{\Omega}$. Clearly, the countable set of polynomials with rational coefficients is a subset of $\mathcal{D}$ and, of course, separates points on $\bar{\Omega}$.

Remark 2.17. In the proof of Proposition 2.16 we see that it is very useful to have compact $\bar{\Omega}$. In this case we can simply choose $\left(F_{k}\right)_{k \in \mathbb{N}}$ as the $\mathcal{E}^{\varrho, a}$-nest consisting of compact sets $F_{k}=\bar{\Omega}$ for all $k \in \mathbb{N}$. Moreover, one can show that, when replacing $\bar{\Omega}$ by an open subset of $\mathbb{R}^{n}$, the corresponding Dirichlet form is not quasi-regular, even in the case when $\varrho=1$ and $a$ is the identity matrix (see [10, Example 1.2.3]). Furthermore, from the proof of Proposition 2.16 we can easily conclude that $\left(\mathcal{E}^{\varrho, a}, D\left(\mathcal{E}^{\varrho, a}\right)\right)$ is even regular (see, for example, $[\mathbf{1 0}]$ ).

\subsection{Locality of the quasi-regular Dirichlet form $\left(\mathcal{E}^{\varrho, a}, D\left(\mathcal{E}^{\varrho, a}\right)\right)$}

A useful property of a Dirichlet form is its so-called locality.

Definition 2.18. A Dirichlet form $(\mathcal{E}, D(\mathcal{E}))$ is said to be local if $\mathcal{E}(u, v)=0$ for all $u, v \in D(\mathcal{E})$ with $\operatorname{supp}(u) \cap \operatorname{supp}(v)=\emptyset$ and $\operatorname{supp}(u), \operatorname{supp}(v)$ are compact.

Proposition 2.19. Suppose that Condition 2.2 is satisfied. Then $\left(\mathcal{E}^{\varrho, a}, D\left(\mathcal{E}^{\varrho, a}\right)\right)$ is local.

Proof. By [20, Chapter V, Example 1.12 (ii)] it is sufficient to show that $\mathcal{D}$ is closed under multiplication and that for the weak gradient we have a product rule. Let $f, g \in \mathcal{D}$. Then obviously $f \cdot g$ is continuous on $\bar{\Omega}$. Furthermore, since $f$ and $g$ are bounded with weak derivatives in $L_{\mathrm{loc}}^{1}(\Omega, \mathrm{d} x), f \cdot g$ is also weakly differentiable and $\nabla(f \cdot g)$ is in $L_{\text {loc }}^{1}(\Omega, \mathrm{d} x)$. Furthermore, the product rule holds and

$$
\nabla(f \cdot g)=\nabla f \cdot g+f \cdot \nabla g, \quad f, g \in \mathcal{D} .
$$

Obviously, $\nabla(f \cdot g) \in L^{2}(\bar{\Omega}, \mu)$ and therefore $\mathcal{E}^{\varrho, a}(f \cdot g, f \cdot g)<\infty$, by ellipticity of $a$.

Let us summarize the properties of the bilinear form $\left(\mathcal{E}^{\varrho, a}, D\left(\mathcal{E}^{\varrho, a}\right)\right)$.

Corollary 2.20. Suppose that Condition 2.2 is satisfied. Then $\left(\mathcal{E}^{\varrho, a}, D\left(\mathcal{E}^{\varrho, a}\right)\right)$ is a conservative, local, quasi-regular Dirichlet form. 
Proof. This follows directly from Propositions 2.6, 2.11, 2.16 and 2.19 and Remark 2.7 (iv).

With these properties we are given an associated Markov process.

Theorem 2.21. Suppose that Condition 2.2 is satisfied. We then have the following results.

(i) There exists a conservative diffusion process (i.e. a Markov process with continuous sample paths and infinite lifetime)

$$
\boldsymbol{M}^{\varrho, a}=\left(\boldsymbol{\Omega}, \boldsymbol{F},\left(\boldsymbol{F}_{t}\right)_{t \geqslant 0},\left(\boldsymbol{\Theta}_{t}\right)_{t \geqslant 0},\left(\boldsymbol{X}_{t}\right)_{t \geqslant 0},\left(\boldsymbol{P}_{x}^{\varrho, a}\right)_{x \in \bar{\Omega}}\right)
$$

with state space $\bar{\Omega}$ which is properly associated with $\left(\mathcal{E}^{\varrho, a}, D\left(\mathcal{E}^{\varrho, a}\right)\right)$, i.e. for all ( $\mu$-versions of) $f \in L^{2}(\bar{\Omega}, \mu)$ and all $t>0$ the function

$$
x \mapsto \int_{\Omega} f\left(\boldsymbol{X}_{t}\right) \mathrm{d} \boldsymbol{P}_{x}^{\varrho, a}, \quad x \in \bar{\Omega},
$$

is an $\mathcal{E}^{\varrho, a}$-quasi-continuous version of $T^{\varrho, a} f . M^{\varrho, a}$ is unique up to $\mu$-equivalence. In particular, $\boldsymbol{M}^{\varrho, a}$ is $\mu$-symmetric (i.e. $\int g T_{t}^{\varrho, a} f \mathrm{~d} \mu=\int f T_{t}^{\varrho, a} g \mathrm{~d} \mu$ for all $f, g: \bar{\Omega} \rightarrow$ $[0, \infty)$ measurable) and has $\mu$ as an invariant measure.

(ii) The diffusion process $\boldsymbol{M}^{\varrho, a}$ is, up to $\mu$-equivalence, the unique diffusion process having $\mu$ as symmetrizing measure and solving the martingale problem for $\left(L^{\varrho, a}, D\left(L^{\varrho, a}\right)\right)$, i.e. for all $g \in D\left(L^{\varrho, a}\right)$,

$$
g\left(\boldsymbol{X}_{t}\right)-g\left(\boldsymbol{X}_{0}\right)-\int_{0}^{t} L^{\varrho, a} g\left(\boldsymbol{X}_{s}\right) \mathrm{d} s, \quad t \geqslant 0,
$$

is an $\boldsymbol{F}_{t}$-martingale under $\boldsymbol{P}_{x}^{o, a}$ (hence starting in $x$ ) for $\mathcal{E}^{\varrho}$-quasi all $x \in \bar{\Omega}$.

In the above theorem $\boldsymbol{M}^{\varrho, a}$ is canonical, i.e. $\boldsymbol{\Omega}=C([0, \infty) \rightarrow \bar{\Omega}), \boldsymbol{X}_{t}(\omega)=\omega(t)$, $\omega \in \boldsymbol{\Omega}$. The filtration $\left(\boldsymbol{F}_{t}\right)_{t \geqslant 0}$ is the natural 'minimum completed admissible filtration' (see [13, Chapter A.2] or [20, Chapter IV]) obtained from the $\sigma$-algebras

$$
\sigma\{\omega(s) \mid 0 \leqslant s \leqslant t, \omega \in \Omega\}, \quad t \geqslant 0 .
$$

$\boldsymbol{F}:=\boldsymbol{F}_{\infty}:=\bigvee_{t \in[0, \infty)} \boldsymbol{F}_{t}$ is the smallest $\sigma$-algebra containing all $\boldsymbol{F}_{t}$, and $\left(\boldsymbol{\Theta}_{t}\right)_{t \geqslant 0}$ are the corresponding natural time shifts. For a detailed discussion of these objects we refer the reader to $[\mathbf{2 0}]$.

Proof. (i) The proof follows directly from [20, Chapter V, Theorem 1.11], since we have already shown that $\left(\mathcal{E}^{\varrho, a}, D\left(\mathcal{E}^{\varrho, a}\right)\right)$ is a conservative, local, quasi-regular Dirichlet form on $L^{2}(\bar{\Omega}, \mu)$.

(ii) Since $\left(\mathcal{E}^{\varrho, a}, D\left(\mathcal{E}^{\varrho, a}\right)\right)$ is a quasi-regular Dirichlet form, the statement follows from [1, Theorem 3.4(i)]. 


\section{The generator of the Dirichlet form $\left(\mathcal{E}^{\varrho, a}, D\left(\mathcal{E}^{\varrho, a}\right)\right)$}

In the previous section we showed that $\left(\mathcal{E}^{\varrho, a}, D\left(\mathcal{E}^{\varrho, a}\right)\right)$ is a Dirichlet form. Thus, the existence of the associated generator $\left(L^{\varrho, a}, D\left(L^{\varrho, a}\right)\right)$ is already clear (see Remark 2.8). In this section we derive an explicit representation of $L^{\varrho, a}$ for certain subsets of its domain $D\left(L^{\varrho, a}\right)$. This representation will be obtained by using the Gaussian integral formula (see, for example, $[\mathbf{4}, \S \mathrm{A} 6.8$, Item (1)]). However, before doing so, we must impose some additional restrictions on the density function $\varrho$ and matrix $a$.

Condition 3.1. We assume that $\sqrt{\varrho} \in W^{1,2}(\Omega)$ and $a \in W^{1, \infty}(\Omega)$.

Here $W^{1,2}(\Omega)$ is the Sobolev space of weakly differentiable, square-integrable functions and $W^{m, \infty}(\Omega), m \in \mathbb{N}$, is the Sobolev space of $m$-times weakly differentiable, essentially bounded functions on $\Omega$. By Sobolev's embedding theorem (see, for example, [4, $\S 8.13]$ ), we have $W^{m, \infty}(\Omega) \subset C^{1}(\bar{\Omega})$ for $m>1$.

Theorem 3.2. Let $\Omega$ have a Lipschitz boundary and let Conditions 2.2 and 3.1 be satisfied. Then

$$
\mathcal{D}_{\mathrm{N}}:=\left\{f \in W^{2, \infty}(\Omega) \mid \partial_{a \nu} f(x)=0 \text { for all } x \in \partial \Omega\right\} \subset D\left(L^{\varrho, a}\right)
$$

and we have the representation

$$
L^{\varrho, a} f=\sum_{i, j=1}^{n} \partial_{i}\left(a_{i j} \partial_{j} f\right)+\partial_{i}(\ln \varrho) a_{i j} \partial_{j} f
$$

(here $\nu$ denotes the outer normal with respect to $\partial \Omega$ and $a \nu$ is the linear transformation of $\nu$ under $a$ ).

Remark 3.3. Now, using Itô's formula, from Theorems 2.21 (ii) and 3.2 we can conclude that the process $\boldsymbol{M}^{o, a}$ solves the stochastic differential equation

$$
\mathrm{d} \boldsymbol{X}_{t}=b\left(\boldsymbol{X}_{t}\right) \mathrm{d} t+\sqrt{2 a}\left(\boldsymbol{X}_{t}\right) \mathrm{d} \boldsymbol{B}_{t}, \quad \text { with reflecting boundary condition, }
$$

inside $\Omega$, for $\mathcal{E}^{\varrho, a}$-quasi all initial conditions $\boldsymbol{X}_{0} \in \bar{\Omega}$. Here, a solution is understood in the sense of the associated martingale problem, $\left(\boldsymbol{B}_{t}\right)_{t \geqslant 0}$ is a vector-valued Brownian motion and

$$
b=\left(\sum_{i=1}^{n} \partial_{i}(\log \varrho) a_{i j}\right)_{1 \leqslant j \leqslant n} .
$$

Proof of Theorem 3.2. In [20, Proposition 2.16] a characterization of the domain of the operator $L^{\varrho, a}$ is given. Namely,

$$
\begin{aligned}
D\left(L^{\varrho, a}\right)=\left\{u \in D\left(\mathcal{E}^{\varrho, a}\right) \mid\right. & v \mapsto \mathcal{E}^{\varrho, a}(u, v) \\
& \text { is continuous with respect to } \left.\sqrt{(\cdot, \cdot)_{L^{2}(\bar{\Omega}, \mu)}} \text { on } D\left(\mathcal{E}^{\varrho, a}\right)\right\} .
\end{aligned}
$$


Therefore, we have to check that the linear operator

$$
A_{f}: D\left(\mathcal{E}^{\varrho, a}\right) \rightarrow \mathbb{R}, \quad g \mapsto \int_{\Omega} \sum_{i, j=1}^{n} \partial_{i} f a_{i j} \partial_{j} g \mathrm{~d} \mu
$$

is continuous with respect to the norm of $L^{2}(\bar{\Omega}, \mu)$ for $f \in \mathcal{D}_{\mathrm{N}}$. Since we can write $\nabla \varrho=\nabla(\sqrt{\varrho} \cdot \sqrt{\varrho})$ and $\varrho$ satisfies Condition 3.1, by the product rule for Sobolev functions we have that $\varrho \in W^{1,1}(\Omega)$. Since $f \in W^{2, \infty}(\Omega)$ and $a \in W^{1, \infty}(\Omega)$, this implies that $u:=\varrho g a_{i j} \partial_{j} f \in W^{1,1}(\Omega)$ for all $g \in \mathcal{D}$ and $i, j \in\{1, \ldots, n\}$. Thus, we can apply the Gaussian integral formula (see, for example, $[4, \S$ A6.8, Item (1)]), and obtain

$$
\int_{\Omega} \partial_{i} u \mathrm{~d} x=\int_{\partial \Omega} u \nu_{i} \mathrm{~d} \mathcal{H}^{n-1}
$$

where $\mathcal{H}^{n-1}$ is the $(n-1)$-dimensional Hausdorff measure on $\partial \Omega$ and $\nu$ the outer normal with respect to $\partial \Omega$. Hence,

$$
\sum_{i, j=1}^{n} \int_{\Omega} \partial_{i}\left(\varrho g a_{i j} \partial_{j} f\right) \mathrm{d} x=0
$$

because

$$
\begin{aligned}
\sum_{i, j=1}^{n} \int_{\partial \Omega}\left(\varrho g a_{i j} \partial_{j} f\right) \nu_{i} \mathrm{~d} \mathcal{H}^{n-1} & =\int_{\partial \Omega}\left(\sum_{i, j=1}^{n} \partial_{j} f a_{i j} \nu_{i}\right) g \varrho \mathrm{d} \mathcal{H}^{n-1} \\
& =\int_{\partial \Omega} \partial_{a \nu} f g \varrho \mathrm{d} \mathcal{H}^{n-1}=0 .
\end{aligned}
$$

Applying the product rule for Sobolev functions to (3.2) and rearranging terms, we obtain

$$
\sum_{i, j=1}^{n} \int_{\Omega} \partial_{j} f a_{i j} \partial_{i} g \mathrm{~d} \mu=-\sum_{i, j=1}^{n} \int_{\Omega}\left(\partial_{i}\left(a_{i j} \partial_{j} f\right)+\partial_{i} \varrho \varrho^{-1} a_{i j} \partial_{j} f\right) g \mathrm{~d} \mu .
$$

Since $\mathcal{D}$ is dense in $\mathcal{D}\left(\mathcal{E}^{\varrho, a}\right)$ with respect to $\sqrt{\mathcal{E}_{1}^{\varrho, a}}$ and $\partial_{i} \varrho \varrho^{-1} \in L^{2}(\bar{\Omega}, \mu)$, we can extend (3.3) to all $g \in \mathcal{D}\left(\mathcal{E}^{\varrho, a}\right)$. To show continuity let us estimate

$$
\begin{aligned}
\left|\sum_{i, j=1}^{n} \int_{\Omega} \partial_{i} f a_{i j} \partial_{j} g \mathrm{~d} \mu\right| \\
\quad \leqslant \kappa \sum_{i, j=1}^{n}\left(\sqrt{\int_{\Omega}\left(\partial_{i} \partial_{j} f\right)^{2} \mathrm{~d} \mu}+\sqrt{\int_{\Omega}\left(\partial_{i} \varrho \varrho^{-1} \partial_{j} f\right)^{2} \mathrm{~d} \mu}\right) \sqrt{\int_{\Omega} g^{2} \mathrm{~d} \mu} \\
\quad=\kappa \sum_{i, j=1}^{n}\left(\sqrt{\int_{\Omega}\left(\partial_{i} \partial_{j} f\right)^{2} \mathrm{~d} \mu}+\sqrt{\int_{\Omega}\left(\frac{\partial_{i} \varrho}{\sqrt{\varrho}} \partial_{j} f\right)^{2} \mathrm{~d} x}\right) \sqrt{\int_{\Omega} g^{2} \mathrm{~d} \mu} \\
\quad=\kappa \sum_{i, j=1}^{n}\left(\sqrt{\int_{\Omega}\left(\partial_{i} \partial_{j} f\right)^{2} \mathrm{~d} \mu}+\sqrt[2]{\int_{\Omega}\left(\partial_{i} \sqrt{\varrho} \partial_{j} f\right)^{2} \mathrm{~d} x}\right) \sqrt{\int_{\Omega} g^{2} \mathrm{~d} \mu}
\end{aligned}
$$


where we have used the ellipticity of $a$. Due to our assumptions on $\varrho$ and $f$, the integrals in (3.4) are finite. Hence, for $f \in \mathcal{D}_{\mathrm{N}}$ the operator $A_{f}$ is continuous and

$$
\mathcal{E}^{\varrho, a}(f, g)=\sum_{i, j=1}^{n} \int_{\Omega} \partial_{i} f a_{i j} \partial_{j} g \varrho \mathrm{d} x=\sum_{i, j=1}^{n} \int_{\Omega}-\left(\partial_{i}\left(a_{i j} \partial_{j} f\right)+\partial_{i} \varrho \varrho^{-1} a_{i j} \partial_{j} f\right) g \mathrm{~d} \mu
$$

for all $f \in \mathcal{D}_{\mathrm{N}}$ and $g \in D\left(\mathcal{E}^{\varrho, a}\right)$. Therefore, for all $f \in \mathcal{D}_{\mathrm{N}}$, the generator $L^{\varrho, a}$ is given by

$$
L^{\varrho, a} f=\sum_{i, j=1}^{n} \partial_{i}\left(a_{i j} \partial_{j} f\right)+\partial_{i} \varrho \varrho^{-1} a_{i j} \partial_{j} f .
$$

\section{Remark 3.4.}

(i) We stress that in $\mathcal{D}_{\mathrm{N}}$ only the normal derivative of the function $f$ is forced to be zero at the boundary. The function $f$ itself is allowed to take arbitrary values at the boundary, i.e. we have Neumann boundary conditions.

(ii) In the proof of Theorem 3.2 we need an $L^{2}$-bound of $\partial_{i} \varrho \varrho^{-1}$. Note that for $\varrho \in L^{1}(\bar{\Omega}, \mathrm{d} x)$ (as we assume anyway) an equivalent condition to Condition 3.1 is $\nabla \ln \varrho \in L^{2}(\bar{\Omega}, \mu)$.

(iii) Obviously, we get the representation of $L^{\varrho, a}$ as in (3.1) for $f \in C_{0}^{\infty}(\Omega)$ without assuming that $\Omega$ has a Lipschitz boundary.

\section{Some potential theory of Dirichlet forms and its consequences}

In this section we show that the set $\{\varrho=0\}:=\{x \in \bar{\Omega} \mid \varrho(x)=0\}$ has capacity zero. As a consequence we can construct the associated process in $\{\varrho>0\}:=\{x \in \bar{\Omega} \mid \varrho(x)>0\}$. This is very important for our construction of the $N$-particle stochastic dynamics with singular interactions (see Remark 5.5).

Definition 4.1. Let $(\mathcal{E}, D(\mathcal{E}))$ be a Dirichlet form on $L^{2}(\bar{\Omega}, \mu)$. The $\mathcal{E}$-capacity $\operatorname{cap}_{\mathcal{E}}(A)$ of an open set $A \subset \bar{\Omega}$ (here, open has to be understood with respect to the trace topology on $\bar{\Omega})$ with respect to $(\mathcal{E}, D(\mathcal{E}))$ is defined by

$$
\operatorname{cap}_{\mathcal{E}}(A)=\inf \left\{\mathcal{E}_{1}(f, f) \mid f \in \mathcal{D}(\mathcal{E}), f \geqslant 1 \mu \text {-a.e. on } A\right\},
$$

and for an arbitrary set $A \subset \bar{\Omega}$ by

$$
\operatorname{cap}_{\mathcal{E}}(A)=\inf \left\{\operatorname{cap}_{\mathcal{E}}(B) \mid B \text { open, } B \supset A\right\} .
$$

For later use we state the following lemma, proved in [10, Theorem 3.1.1].

Lemma 4.2. Let $A_{m}, m \in \mathbb{N}$, be an increasing sequence of subsets of $\bar{\Omega}$. Then

$$
\operatorname{cap}_{\mathcal{E}}\left(\bigcup_{m \in \mathbb{N}} A_{m}\right)=\sup _{m \in \mathbb{N}} \operatorname{cap}_{\mathcal{E}}\left(A_{m}\right)
$$


To make use of capacity estimates provided in $[\mathbf{2 2}]$ we need the next definition and the following lemma.

Definition 4.3. In our situation a regular Dirichlet form $(\mathcal{E}, D(\mathcal{E}))$ with $D(\mathcal{E}) \subset$ $L^{2}(\bar{\Omega}, \mu)$ is called strongly regular if the topology induced by the intrinsic metric

$$
\begin{aligned}
d(x, y):=\sup \{u(x)-u(y) \mid u \in & D(\mathcal{E}) \cap C(\bar{\Omega}) \\
& \left.\quad \text { with } \sum_{i, j=1}^{n} a_{i j} \partial_{i} u \partial_{j} u \varrho \leqslant \varrho \text { a.e. on } \bar{\Omega}\right\}, \quad x, y \in \bar{\Omega},
\end{aligned}
$$

coincides with the topology generated by the Euclidean metric.

Lemma 4.4. $\left(\mathcal{E}^{\varrho, a}, D\left(\mathcal{E}^{\varrho, a}\right)\right)$ is strongly regular.

Proof. The intrinsic metric of the underlying Dirichlet form $\left(\mathcal{E}^{\varrho, a}, D\left(\mathcal{E}^{\varrho}, a\right)\right)$ is given by

$$
\begin{aligned}
d(x, y):=\sup \left\{u(x)-u(y) \mid u \in D\left(\mathcal{E}^{\varrho, a}\right) \cap C(\bar{\Omega})\right. \\
\left.\quad \text { with } \sum_{i, j=1}^{n} a_{i j} \partial_{i} u \partial_{j} u \varrho \leqslant \varrho \text { a.e. on } \bar{\Omega}\right\} \quad \text { for } x, y \in \bar{\Omega} .
\end{aligned}
$$

By assumption we have $\varrho>0$ a.e. on $\bar{\Omega}$. Thus,

$$
d(x, y)=\sup \left\{u(x)-u(y) \mid u \in D\left(\mathcal{E}^{\varrho, a}\right) \cap C(\bar{\Omega}) \text { with } \sum_{i, j=1}^{n} a_{i j} \partial_{i} u \partial_{j} u \leqslant 1 \text { a.e. on } \bar{\Omega}\right\}
$$

for $x, y \in \bar{\Omega}$. Since $\Omega$ is convex, it follows easily by the fundamental theorem of calculus that

$$
d_{\mathrm{E}}(x, y)=\sup \left\{u(x)-u(y) \mid u \in D\left(\mathcal{E}^{\varrho, a}\right) \cap C(\bar{\Omega}) \text { with }|\nabla u|^{2} \leqslant 1 \text { a.e. on } \bar{\Omega}\right\}
$$

for $x, y \in \bar{\Omega}$, where $d_{\mathrm{E}}$ is the metric induced by the Euclidean norm on $\mathbb{R}^{n}$. By the ellipticity of $a$ we have

$$
\kappa^{-1}|\nabla u|^{2} \leqslant \sum_{i, j=1}^{n} a_{i j} \partial_{i} u \partial_{j} u \leqslant \kappa|\nabla u|^{2} \quad \text { for all } u \in D\left(\mathcal{E}^{\varrho, a}\right) \cap C(\bar{\Omega}) .
$$

Hence,

$$
\begin{aligned}
&\left\{u \in D\left(\mathcal{E}^{\varrho, a}\right) \cap C(\bar{\Omega}) \mid \sum_{i, j=1}^{n} a_{i j} \partial_{i} u \partial_{j} u\right.\leqslant 1 \text { a.e. on } \bar{\Omega}\} \\
& \supset\left\{\left.u \in D\left(\mathcal{E}^{\varrho, a}\right) \cap C(\bar{\Omega})|\kappa| \nabla u\right|^{2} \leqslant 1 \text { a.e. on } \bar{\Omega}\right\}
\end{aligned}
$$

and we obtain

$$
d(x, y) \geqslant \kappa^{-1 / 2} d_{\mathrm{E}}(x, y) \quad \text { for } x, y \in \bar{\Omega} .
$$


An argument analogous to that above yields $d(x, y) \leqslant \kappa^{1 / 2} d_{\mathrm{E}}(x, y)$ for $x, y \in \bar{\Omega}$. Therefore, the intrinsic metric of the underlying Dirichlet form $\left(\mathcal{E}^{\varrho, a}, D\left(\mathcal{E}^{\varrho, a}\right)\right)$ is equivalent to the Euclidean metric. Hence, the topologies generated by them coincide, i.e. $\left(\mathcal{E}^{\varrho, a}, D\left(\mathcal{E}^{\varrho, a}\right)\right)$ is strongly regular.

We are now ready to state and prove the main result of this section.

Theorem 4.5. Suppose that the density function $\varrho$ satisfies Condition 2.2. Furthermore, assume that one of the following two conditions holds.

(i) @ satisfies Condition 3.1 and is continuous on $\bar{\Omega}$.

(ii) $\Omega$ is convex and there exists $0<C<\infty$ such that

$$
\int_{B_{r}(\{\varrho=0\})} \varrho(x) \mathrm{d} x \leqslant C r^{2} \quad \text { as } r \rightarrow 0 .
$$

Then

$$
\operatorname{cap}^{\varrho, a}(\{\varrho=0\})=0
$$

where $\operatorname{cap}^{\varrho, a}:=\operatorname{cap}_{\mathcal{E}^{\varrho, a}}$

Proof. In (i) we know that $\psi:=\sqrt{\varrho}>0 \mathrm{~d} x$-a.e. and $\psi \in W^{1,2}(\Omega)$. For $\varepsilon>0$ let $\psi_{\varepsilon}:=(\psi \vee \varepsilon) \wedge 1$ and $f_{\varepsilon}=-\log \left(\psi_{\varepsilon}\right)$. Then $f_{\varepsilon}$ is continuous on $\bar{\Omega}, \nabla f_{\varepsilon}=-\nabla \psi_{\varepsilon} / \psi_{\varepsilon}$ and

$$
\left(\nabla f_{\varepsilon}, \nabla f_{\varepsilon}\right)_{\mathbb{R}^{n}}=\left(\nabla \psi_{\varepsilon}, \nabla \psi_{\varepsilon}\right)_{\mathbb{R}^{n}} \psi_{\varepsilon}^{-2} \leqslant\left(\nabla \psi_{\varepsilon}, \nabla \psi_{\varepsilon}\right)_{\mathbb{R}^{n}} \varrho^{-1} \in L^{1}(\bar{\Omega}, \mathrm{d} \mu),
$$

since $\psi_{\varepsilon} \in W^{1,2}(\Omega)$. Thus, $f_{\varepsilon} \in \mathcal{D}\left(\mathcal{E}^{\varrho, a}\right)$. We have

$$
\begin{aligned}
\mathcal{E}_{1}^{\varrho, a}\left(f_{\varepsilon}, f_{\varepsilon}\right) & =\sum_{i, j=1}^{n} \int_{\Omega} \partial_{i} f_{\varepsilon} a_{i j} \partial_{j} f_{\varepsilon} \psi^{2} \mathrm{~d} x+\int_{\bar{\Omega}} f_{\varepsilon}^{2} \mathrm{~d} \mu \\
& =\sum_{i, j=1}^{n} \int_{\Omega} \partial_{i} \psi_{\varepsilon} a_{i j} \partial_{j} \psi_{\varepsilon} \frac{\psi^{2}}{\psi_{\varepsilon}{ }^{2}} \mathrm{~d} x+\int_{\bar{\Omega}} \log \left(\psi_{\varepsilon}\right)^{2} \mathrm{~d} \mu \\
& \leqslant \kappa \sum_{i=1}^{n} \int_{\Omega}\left(\partial_{i} \psi_{\varepsilon}\right)^{2} \mathrm{~d} x+\int_{\bar{\Omega}} \log \left(\psi_{\varepsilon}\right)^{2} \mathrm{~d} \mu<\infty
\end{aligned}
$$

(in view of our assumptions on $\psi$ ). Let $\lambda>0$. Then

$$
\begin{aligned}
\operatorname{cap}^{\varrho, a}\left(\left\{f_{\varepsilon}>\lambda\right\}\right) & \leqslant \mathcal{E}_{1}^{\varrho, a}\left(\frac{1}{\lambda} f_{\varepsilon}, \frac{1}{\lambda} f_{\varepsilon}\right) \\
& \leqslant \frac{\kappa}{\lambda^{2}}\left(\sum_{i=1}^{n} \int_{\Omega}\left(\partial_{i} \psi_{\varepsilon}\right)^{2} \mathrm{~d} x+\int_{\bar{\Omega}} \log \left(\psi_{\varepsilon}\right)^{2} \mathrm{~d} \mu\right)
\end{aligned}
$$

by Definition 4.1 and (4.1). Next we set $\varepsilon=1 / m, m \in \mathbb{N}$, and consider

$$
A_{m}:=\left\{f_{1 / m}>\lambda\right\}
$$


We observe that the sequence of sets $A_{m}$ is increasing in $m \in \mathbb{N}$. Thus, for $A:=\bigcup_{m=1}^{\infty} A_{m}$ Lemma 4.2 yields

$$
\operatorname{cap}^{\varrho, a}(A)=\sup _{m \in \mathbb{N}} \operatorname{cap}^{\varrho, a}\left(A_{m}\right) \leqslant \sup _{m \in \mathbb{N}} \frac{\kappa}{\lambda^{2}}\left(\sum_{i=1}^{n} \int_{\Omega}\left(\partial_{i} \psi_{1 / m}\right)^{2} \mathrm{~d} x+\int_{\bar{\Omega}} \log \left(\psi_{1 / m}\right)^{2} \mathrm{~d} \mu\right) .
$$

Since in the above integrals we are dealing with functions pointwisely monotone increasing in $m \in \mathbb{N}$, the supremum coincides with

$$
\begin{aligned}
\lim _{m \rightarrow \infty} \frac{\kappa}{\lambda^{2}}\left(\sum_{i=1}^{n} \int_{\Omega}\left(\partial_{i} \psi_{1 / m}\right)^{2} \mathrm{~d} x\right. & \left.+\int_{\bar{\Omega}} \log \left(\psi_{1 / m}\right)^{2} \mathrm{~d} \mu\right) \\
& =\frac{\kappa}{\lambda^{2}}\left(\sum_{i=1}^{n} \int_{\Omega}\left(\partial_{i} \psi\right)^{2} \mathrm{~d} x+\int_{\bar{\Omega}} \log (\psi)^{2} \psi^{2} \mathrm{~d} x\right)<\infty
\end{aligned}
$$

due to our assumptions on $\psi$. Observe that $A=\{\log (\psi)>\lambda\}$. Therefore,

$$
\operatorname{cap}^{\varrho, a}(\{\log (\psi)>\lambda\}) \leqslant \frac{\kappa}{\lambda^{2}}\left(\sum_{i=1}^{n} \int_{\Omega}\left(\partial_{i} \psi\right)^{2} \mathrm{~d} x+\int_{\bar{\Omega}} \log (\psi)^{2} \psi^{2} \mathrm{~d} x\right) .
$$

Thus,

$$
\begin{aligned}
\operatorname{cap}^{\varrho, a}(\{\varrho=0\}) & \leqslant \operatorname{cap}^{\varrho, a}(\{\log (\varrho)>2 \lambda\}) \\
& =\operatorname{cap}^{\varrho, a}(\{\log (\psi)>\lambda\}) \\
& \leqslant \frac{\kappa}{\lambda^{2}}\left(\sum_{i=1}^{n} \int_{\Omega}\left(\partial_{i} \psi\right)^{2} \mathrm{~d} x+\int_{\bar{\Omega}} \log (\psi)^{2} \psi^{2} \mathrm{~d} x\right) \rightarrow 0 \quad \text { as } \lambda \rightarrow \infty
\end{aligned}
$$

In (ii), by Lemma 4.4 we have that $\left(\mathcal{E}^{\varrho, a}, D\left(\mathcal{E}^{\varrho}, a\right)\right)$ is strongly regular. By assumption, the compact set $\{\varrho=0\}$ is of $\mu$-measure zero. Thus, we can apply [22, Theorem 3] and the proof follows.

Remark 4.6. Some of the ideas for the proof of Theorem 4.5 (i) were obtained from the proof of [11, Theorem 2].

Let $i:\{\varrho>0\} \rightarrow\{\varrho>0\}$ be the identity map. Since $\{\varrho=0\}=\bar{\Omega} \backslash\{\varrho>0\}$ has Lebesgue measure zero, we can consider the isometry $i^{*}: L^{2}(\{\varrho>0\}, \mu) \rightarrow L^{2}(\bar{\Omega}, \mu)$ by defining $i^{*}(f)$ to be the $\mu$-class represented by $\tilde{f} \circ i$ on $\{\varrho>0\}$ for any measurable $\mu$ version $\tilde{f}$ of $f \in L^{2}(\{\varrho>0\}, \mu)$. Obviously, $i^{*}$ is surjective and, owing to [20, Chapter VI, Exercise 1.1],

$$
\begin{aligned}
\widehat{\mathcal{E} \varrho, a}(f, g) & :=\mathcal{E}^{\varrho, a}\left(i^{*}(f), i^{*}(g)\right), \quad f, g \in D(\widehat{\mathcal{E} \varrho, a}), \\
D(\widehat{\mathcal{E} \varrho, a}) & :=\left\{f \in L^{2}(\{\varrho>0\}, \mu) \mid f \in i^{*-1}\left(D\left(\mathcal{E}^{\varrho, a}\right)\right)\right\}
\end{aligned}
$$

is a Dirichlet form on $L^{2}(\{\varrho>0\}, \mu) \cdot(\widehat{\mathcal{E} \varrho, a}, D(\widehat{\mathcal{E} \varrho, a}))$ is called the image Dirichlet form of $\left(\mathcal{E}^{\varrho, a}, D\left(\mathcal{E}^{\varrho, a}\right)\right)$ under $i$. 
Corollary 4.7. Under the same assumptions as made in Theorem 4.5, $(\widehat{\mathcal{E} \varrho, a}, D(\widehat{\mathcal{E} \varrho, a}))$ is a conservative, local, quasi-regular Dirichlet form on $L^{2}(\{\varrho>0\}, \mu)$.

Proof. By Theorem 4.5 we know that $\operatorname{cap}^{\varrho, a}(\{\varrho=0\})=0$ and this is equivalent to the fact that $\{\varrho=0\}$ is an $\mathcal{E}^{\varrho, a}$-exceptional set, i.e.

$$
\{\varrho=0\} \subset \bigcap_{k \geqslant 1} F_{k}^{\mathrm{c}} \quad \text { for some } \mathcal{E}^{\varrho, a} \text {-nest }\left(F_{k}\right)_{k \geqslant 1}
$$

(see [3, Proposition $14(3)]$ ). Thus, $\left(F_{k}\right)_{k \in \mathbb{N}}$ is a sequence of compact sets in $\{\varrho>0\}$. Note that functions from $D(\widehat{\mathcal{E} Q, a})$ are the restrictions to $\{\varrho>0\}$ of functions from $D\left(\mathcal{E}^{\varrho, a}\right)$. Since $\bigcup_{k \geqslant 1} D\left(\mathcal{E}^{\varrho, a}\right)_{F_{k}}$ is a dense set in $D\left(\mathcal{E}^{\varrho, a}\right), \bigcup_{k \geqslant 1} D(\widehat{\mathcal{E} \varrho, a})_{F_{k}}$ is a dense set in $D(\widehat{\mathcal{E} Q, a})$. Hence, $(\widehat{\mathcal{E} Q, a}, D(\widehat{\mathcal{E} Q, a}))$ has a compact $\widehat{\mathcal{E} Q, a}$-nest. Furthermore, the functions from $\mathcal{D}$ restricted to $\{\varrho>0\} \subset \bar{\Omega}$ again are continuous functions. Now, since $D\left(\mathcal{E}^{o, a}\right)$ is the completion of $\mathcal{D}$ with respect to $\sqrt{ } \mathcal{E}_{1}^{\varrho, a}$ in $L^{2}(\bar{\Omega}, \mu)$ and $\{\varrho=0\}$ is of Lebesgue measure zero, the continuous functions in $D(\widehat{\mathcal{E} \varrho, a})$ are dense in $D(\widehat{\mathcal{E} Q, a})$ with respect to $\sqrt{\mathcal{E}_{1}^{\varrho, a}}$. Clearly, the countable set of polynomials with rational coefficients is separating points on $\{\varrho>0\}$. Locality and conservativity are implied by locality and conservativity of $\left(\mathcal{E}^{\varrho, a}, D\left(\mathcal{E}^{\varrho, a}\right)\right)$ (see Definition 2.18 and Remark 2.7 (iv)).

We denote the generator of $(\widehat{\mathcal{E} \varrho, a}, D(\widehat{\mathcal{E} \varrho, a}))$ by $\left(\widehat{L^{\varrho, a}}, D\left(\widehat{L^{\varrho, a}}\right)\right)$. The strongly continuous contraction semigroup generated by $\left(\widehat{L^{\varrho, a}}, D\left(\widehat{L^{\varrho, a}}\right)\right)$ is denoted by $\left(\widehat{T^{\varrho, a}} t\right)_{t \geqslant 0}$. We then have the following corollary.

Corollary 4.8. Under the assumptions in Theorem 4.5 there exists a conservative diffusion process $\widehat{M^{\varrho, a}}$ in $\{\varrho>0\}$ associated with the Dirichlet form $(\widehat{\mathcal{E} \varrho, a}, D(\widehat{\mathcal{E} \varrho, a}))$. Furthermore, all statements of Theorem 2.21 hold true if $\left(\mathcal{E}^{\varrho, a}, D\left(\mathcal{E}^{\varrho, a}\right)\right),\left(L^{\varrho, a}, D\left(L^{\varrho, a}\right)\right)$, $\left(T_{t}^{\varrho, a}\right)_{t \geqslant 0}$ and $\boldsymbol{M}^{\varrho, a}$ are replaced by $(\widehat{\mathcal{E} \varrho, a}, D(\widehat{\mathcal{E} \varrho, a})),\left(\widehat{L^{\varrho, a}}, D\left(\widehat{L^{\varrho, a}}\right)\right),\left(\widehat{T^{\varrho, a}} t\right)_{t \geqslant 0}$ and $\widehat{\boldsymbol{M}^{\varrho, a}}$, respectively.

\section{An application to continuous $N$-particle systems with singular interactions}

Let $d \in \mathbb{N}$. A pair potential (without hard core) is a Borel measurable function $\phi: \mathbb{R}^{d} \rightarrow$ $\mathbb{R} \cup\{\infty\}$ such that $\phi(-x)=\phi(x) \in \mathbb{R}$ for all $x \in \mathbb{R}^{d} \backslash\{0\}$. Let us fix our assumptions on the potential $\phi$.

(RP) Repulsion: there exists a continuous decreasing function $\Phi:(0, \infty) \rightarrow[0, \infty)$ with $\lim _{t \rightarrow 0} \Phi(t)=\infty$ and $R_{1}>0$ such that

$$
\phi(x) \geqslant \Phi(|x|) \text { for }|x| \leqslant R_{1} .
$$

Furthermore, the potential is bounded from above on

$$
\left\{x \in \mathbb{R}^{d}|\kappa \leqslant| x \mid\right\} \text { for all } \kappa>0 .
$$


(SRP) Strong repulsion: there exists $R_{1}>0$ such that

$$
\phi(x) \geqslant-\ln (|x|) \quad \text { for } 0<|x| \leqslant R_{1} .
$$

Furthermore, the potential is bounded from above on

$$
\left\{x \in \mathbb{R}^{d}|\kappa \leqslant| x \mid\right\} \text { for all } \kappa>0 .
$$

(BB) Bounded below: there exists $0 \leqslant B<\infty$ such that

$$
\phi(x) \geqslant-B \text { for all } x \in \mathbb{R}^{d} .
$$

$\left(\mathrm{DL}^{2}\right)$ Differentiability and $L^{2}$ : the function $\exp (-\phi)$ is weakly differentiable on $\mathbb{R}^{d}, \phi$ is continuous on $\mathbb{R}^{d} \backslash\{0\}$ and weakly differentiable on $\mathbb{R}^{d}$. The gradient $\nabla \phi$, considered as a $\mathrm{d} x$-a.e. defined function on $\mathbb{R}^{d}$, satisfies

$$
\nabla \phi \in L_{\mathrm{loc}}^{2}\left(\mathbb{R}^{d}, \exp (-\phi) \mathrm{d} x\right) .
$$

Remark 5.1. Note that for many typical potentials in statistical physics we have $\phi \in C^{\infty}\left(\mathbb{R}^{d} \backslash\{0\}\right)$. For such 'outside the origin regular' potentials, condition ( $\left.\mathrm{DL}^{2}\right)$ nevertheless does not exclude a singularity at the point $0 \in \mathbb{R}^{d}$.

Let $N, d \in \mathbb{N}$ and $\Lambda \subset \mathbb{R}^{d}$, such that $\bar{\Omega}:=\Lambda^{N} \subset \mathbb{R}^{N \cdot d}$ is the closure of an open, relatively compact set having boundary $\partial\left(\Lambda^{N}\right)$ of Lebesgue measure zero. On $\Lambda^{N}$, we consider the density function

$$
\varrho_{\Lambda, N}(x)=\frac{1}{Z_{\Lambda, N}} \exp \left(-\sum_{1 \leqslant i<j \leqslant N} \phi\left(x_{i}-x_{j}\right)\right), \quad x=\left(x_{1}, \ldots, x_{N}\right) \in \Lambda^{N},
$$

where

$$
Z_{\Lambda, N}:=\int_{\Lambda^{N}} \exp \left(-\sum_{1 \leqslant i<j \leqslant N} \phi\left(x_{i}-x_{j}\right)\right) \mathrm{d} x^{\otimes N}
$$

Proposition 5.2. If $d=1$, we suppose that either

(A) $\phi$ satisfies conditions (SRP) and (BB) and $\Lambda$ is convex, or

(B) $\phi$ satisfies conditions $(R P)$ and $\left(D L^{2}\right)$.

If $d \geqslant 2$, we suppose that either

(C) $\phi$ satisfies conditions $(R P)$ and $(B B)$ and $\Lambda$ is convex, or

(D) $\phi$ is bounded and $\Lambda$ is convex.

Then in all situations $\left(\mathcal{E}_{\Lambda, N}, \mathcal{D}\right)$ is closable. Its closure $\left(\mathcal{E}_{\Lambda, N}, D\left(\mathcal{E}_{\Lambda, N}\right)\right)$ is a conservative, local, quasi-regular Dirichlet form on $L^{2}\left(\Lambda^{N}, \mu_{\Lambda, N}\right)$. We denote its generator by $\left(L_{\Lambda, N}, D\left(L_{\Lambda, N}\right)\right)$. 
Proof. In each situation it is easy to check that Condition 2.2 holds. Thus, the proof follows from Corollary 2.20.

As before, Proposition 5.2 now implies the existence of a corresponding conservative diffusion process in $\Lambda^{N} . \Lambda^{N}$, however, has no direct interpretation as a continuous $N$-particle system. This leads us to the configuration space over $\mathbb{R}^{d}$, which is defined as the set of all subsets of $\mathbb{R}^{d}$ which are locally finite,

$$
\Gamma:=\left\{\gamma \subset \mathbb{R}^{d} \mid \#\left(\gamma_{\Lambda}\right)<\infty \text { for each compact } \Lambda \subset \mathbb{R}^{d}\right\},
$$

where \# denotes the number of elements of a set and $\gamma_{\Lambda}:=\gamma \cap \Lambda$. One can identify $\gamma \in \Gamma$ with the positive Radon measure $\sum_{x \in \gamma} \varepsilon_{x} \in \mathcal{M}\left(\mathbb{R}^{d}\right)$, where $\varepsilon_{x}$ is the Dirac measure at $x$, $\sum_{x \in \emptyset} \varepsilon_{x}:=$ zero measure and $\mathcal{M}\left(\mathbb{R}^{d}\right)$ stands for the set of all positive Radon measures on the Borel $\sigma$-algebra $\mathcal{B}\left(\mathbb{R}^{d}\right)$. Hence, via this identification, $\Gamma$ can be assigned the vague topology. We set $\Gamma_{\Lambda}:=\{\gamma \in \Gamma \mid \gamma \subset \Lambda\}$. The space of $N$-point configurations in $\Lambda$ is defined by

$$
\Gamma_{\Lambda}^{(N)}:=\{\gamma \subset \Lambda \mid \#(\gamma)=N\} \subset \Gamma_{\Lambda} \subset \Gamma .
$$

To define more structure on $\Gamma_{\Lambda}^{(N)}$ we may use the natural mapping

$$
\begin{aligned}
\operatorname{sym}^{(N)}: \tilde{\Lambda}^{N} & \rightarrow \Gamma_{\Lambda}^{(N)}, \\
\operatorname{sym}^{(N)}\left(x_{1}, \ldots, x_{N}\right) & :=\left\{x_{1}, \ldots, x_{N}\right\},
\end{aligned}
$$

where

$$
\tilde{\Lambda}^{N}:=\left\{\left(x_{1}, \ldots, x_{N}\right) \in \Lambda^{N} \mid x_{k} \neq x_{j} \text { if } k \neq j\right\} .
$$

We assume these mappings in order to generate the topology and corresponding Borel $\sigma$-algebra $\mathcal{B}\left(\Gamma_{\Lambda}^{(N)}\right)$ on $\Gamma_{\Lambda}^{(N)}$. Obviously, this $\sigma$-algebra coincides with the Borel $\sigma$-algebra inherited from $\Gamma$ equipped with its vague topology. Of course, the product measure $\mathrm{d} x^{\otimes N}$ can be considered on $\tilde{\Lambda}^{N}$. Let $\mathrm{d} x^{(N)}:=\mathrm{d} x^{\otimes N} \circ\left(\operatorname{sym}^{(N)}\right)^{-1}$ denote the corresponding measure on $\Gamma_{\Lambda}^{(N)}$.

In order to construct the $N$-particle stochastic dynamics, we are interested in the image Dirichlet form $\left(\mathcal{E}_{\Lambda}^{(N)}, D\left(\mathcal{E}_{\Lambda}^{(N)}\right)\right)$ of $\left(\mathcal{E}_{\Lambda, N}, D\left(\mathcal{E}_{\Lambda, N}\right)\right)$ under sym ${ }^{(N)}$. Consider the measure

$$
\mu_{\Lambda}^{(N)}:=\mu_{\Lambda, N} \circ\left(\operatorname{sym}^{(N)}\right)^{-1} .
$$

$\mu_{\Lambda}^{(N)}$ is the canonical $N$-particle Gibbs measure in $\Lambda$ with empty boundary conditions on $\left(\Gamma_{\Lambda}^{(N)}, \mathcal{B}\left(\Gamma_{\Lambda}^{(N)}\right)\right)$. Define an isometry

$$
\left(\operatorname{sym}^{(N)}\right)^{*}: L^{2}\left(\Gamma_{\Lambda}^{(N)}, \mu_{\Lambda}^{(N)}\right) \rightarrow L^{2}\left(\Lambda^{N}, \mu_{\Lambda, N}\right)
$$

by setting $\left(\operatorname{sym}^{(N)}\right)^{*} F$ to be the $\mu_{\Lambda, N}$-class represented by $\tilde{F} \circ \operatorname{sym}_{\Lambda}^{(N)}$ on $\tilde{\Lambda}^{N}$ for any $\mu_{\Lambda}^{(N)}$-version $\tilde{F}$ of $F \in L^{2}\left(\Gamma_{\Lambda}^{(N)}, \mu_{\Lambda}^{(N)}\right)$ (note that the set of diagonals $D g:=\Lambda^{N} \backslash \tilde{\Lambda}^{N}$ has $\mu_{\Lambda, N}$-measure zero).

Note that the subspace

$$
L_{\mathrm{sym}}^{2}\left(\Lambda^{N}, \mu_{\Lambda, N}\right):=\left(\operatorname{sym}^{(N)}\right)^{*}\left(L^{2}\left(\Gamma_{\Lambda}^{(N)}, \mu_{\Lambda}^{(N)}\right)\right) \subset L^{2}\left(\Lambda^{N}, \mu_{\Lambda, N}\right)
$$


is the closed subspace of symmetric functions from $L^{2}\left(\Lambda^{N}, \mu_{\Lambda, N}\right)$. Using this mapping one can define a bilinear form $\left(\mathcal{E}_{\Lambda}^{(N)}, D\left(\mathcal{E}_{\Lambda}^{(N)}\right)\right)$ as the image bilinear form of $\left(\mathcal{E}_{\Lambda, N}, D\left(\mathcal{E}_{\Lambda, N}\right)\right)$ under $\operatorname{sym}^{(N)}$ :

$$
\mathcal{E}_{\Lambda}^{(N)}(F, G):=\mathcal{E}_{\Lambda, N}\left(\left(\operatorname{sym}^{(N)}\right)^{*} F,\left(\operatorname{sym}^{(N)}\right)^{*} G\right), \quad F, G \in D\left(\mathcal{E}_{\Lambda}^{(N)}\right),
$$

where

$$
D\left(\mathcal{E}_{\Lambda}^{(N)}\right):=\left(\left(\operatorname{sym}^{(N)}\right)^{*}\right)^{-1}\left(D\left(\mathcal{E}_{\Lambda, N}\right) \cap L_{\mathrm{sym}}^{2}\left(\mu_{\Lambda, N}\right)\right) .
$$

Proposition 5.3. If $d=1$, we suppose that either

(A) $\phi$ satisfies conditions (SRP) and (BB) and $\Lambda$ is convex, or

(B) $\phi$ satisfies conditions $(R P)$ and $\left(D L^{2}\right)$.

If $d \geqslant 2$, we suppose that either

(C) $\phi$ satisfies conditions $(R P)$ and $(B B)$ and $\Lambda$ is convex, or

(D) $\phi$ is bounded and $\Lambda$ is convex.

Then in all situations the bilinear form $\left(\mathcal{E}_{\Lambda}^{(N)}, D\left(\mathcal{E}_{\Lambda}^{(N)}\right)\right)$ is a conservative, local, quasiregular Dirichlet form on $L^{2}\left(\Gamma_{\Lambda}^{(N)}, \mu_{\Lambda}^{(N)}\right)$. Its generator is given by

$$
\begin{aligned}
L_{\Lambda}^{(N)} & =\left(\left(\operatorname{sym}^{(N)}\right)^{*}\right)^{-1} \circ L_{\Lambda, N} \circ\left(\operatorname{sym}^{(N)}\right)^{*}, \\
D\left(L_{\Lambda}^{(N)}\right) & =\left(\left(\operatorname{sym}^{(N)}\right)^{*}\right)^{-1}\left(D\left(L_{\Lambda, N}\right) \cap L_{\text {sym }}^{2}\left(\mu_{\Lambda, N}\right)\right) .
\end{aligned}
$$

Of course, $\left(L_{\Lambda}^{(N)}, D\left(L_{\Lambda}^{(N)}\right)\right)$ generates a strongly continuous contraction semi-group

$$
T_{\Lambda}^{(N)}(t):=\exp \left(t L_{\Lambda}^{(N)}\right), \quad t \geqslant 0 .
$$

Proof. In situations (A) and (C) we have $D g=\left\{\varrho_{\Lambda, N}=0\right\}$ due to conditions (SRP) and $(\mathrm{RP})$, respectively. Since $D g$ has codimension at least $d$, Theorem 4.5 (ii) is satisfied and $\operatorname{cap}_{\mathcal{E}_{\Lambda, N}}(D g)=0$. In situation (B) we also have $D g=\left\{\varrho_{\Lambda, N}=0\right\}$ due to condition $(\mathrm{RP})$. Thus, $\operatorname{cap}_{\mathcal{E}_{\Lambda, N}}(D g)=0$, because Theorem $4.5(\mathrm{i})$ is satisfied. This can be seen by using Remark 3.4 (ii) and a Sobolev embedding. In situation (D) we have $\left\{\varrho_{\Lambda, N}=0\right\}=\emptyset$, since $\phi$ is bounded. $D g=\Lambda^{N} \backslash \tilde{\Lambda}^{N}$ is of codimension at least $d .\left(\mathcal{E}^{\varrho_{\Lambda, N}}, D\left(\mathcal{E}^{\varrho_{\Lambda, N}}\right)\right)$ is strongly regular (as shown in the proof of Lemma 4.4). Thus, due to [22, Theorem 3] we have that $\Lambda^{N} \backslash \tilde{\Lambda}^{N}$ is of capacity zero with respect to $\left(\mathcal{E}^{\varrho_{\Lambda, N}}, D\left(\mathcal{E}^{\varrho_{\Lambda, N}}\right)\right)$. Therefore, as in the proof of Corollary 4.7, we obtain that $\left(\mathcal{E}_{\Lambda}^{(N)}, \mathcal{D}\left(\mathcal{E}_{\Lambda}^{(N)}\right)\right)$ is a conservative, local, quasi-regular Dirichlet form on $L^{2}\left(\Gamma_{\Lambda}^{(N)}, \mu_{\Lambda}^{(N)}\right)$. We merely take sym ${ }^{(N)}$ and $\left(\operatorname{sym}^{(N)}\right)^{*}$ instead of the mappings $i$ and $i^{*}$, respectively, and use the fact that the isometry

$$
\left(\operatorname{sym}^{(N)}\right)^{*}: L^{2}\left(\Gamma_{\Lambda}^{(N)}, \mu_{\Lambda}^{(N)}\right) \rightarrow L_{\text {sym }}^{2}\left(\Lambda^{N}, \mu_{\Lambda, N}\right)
$$

is surjective. 
Theorem 5.4. If $d=1$, we suppose that either

(A) $\phi$ satisfies conditions (SRP) and $(B B)$ and $\Lambda$ is convex, or

(B) $\phi$ satisfies conditions $(R P)$ and $\left(D L^{2}\right)$.

If $d \geqslant 2$, we suppose that either

(C) $\phi$ to satisfies conditions $(R P)$ and $(B B)$ and $\Lambda$ is convex, or

(D) $\phi$ is bounded and $\Lambda$ is convex.

Then, in all situations, we have the following.

(i) There exists a conservative diffusion process (i.e. a strong Markov process with continuous sample paths and infinite lifetime)

$$
\boldsymbol{M}_{\Lambda}^{(N)}=\left(\boldsymbol{\Omega}_{\Lambda}^{(N)}, \boldsymbol{F}_{\Lambda}^{(N)},\left(\boldsymbol{F}_{\Lambda}^{(N)}(t)\right)_{t \geqslant 0},\left(\boldsymbol{\Theta}_{\Lambda}^{(N)}(t)\right)_{t \geqslant 0},(\boldsymbol{X}(t))_{t \geqslant 0},\left(\boldsymbol{P}_{\Lambda}^{(N)}(x)\right)_{x \in \Gamma_{\Lambda}^{(N)}}\right)
$$

in $\Gamma_{\Lambda}^{(N)}$ which is properly associated with $\left(\mathcal{E}_{\Lambda}^{(N)}, D\left(\mathcal{E}_{\Lambda}^{(N)}\right)\right)$, i.e. for all $\left(\mu_{\Lambda}^{(N)}\right.$-versions of) $F \in L^{2}\left(\Gamma_{\Lambda}^{(N)}, \mu_{\Lambda}^{(N)}\right)$ and all $t>0$ the function

$$
x \mapsto \int_{\boldsymbol{\Omega}_{\Lambda}^{(N)}} F(\boldsymbol{X}(t)) \mathrm{d} \boldsymbol{P}_{\Lambda}^{(N)}(x), \quad x \in \Gamma_{\Lambda}^{(N)},
$$

is an $\mathcal{E}_{\Lambda}^{(N)}$-quasi-continuous version of $T_{\Lambda}^{(N)}(t) F . M_{\Lambda}^{(N)}$ is unique up to $\mu_{\Lambda}^{(N)}$-equivalence. In particular, $\boldsymbol{M}_{\Lambda}^{(N)}$ is $\mu_{\Lambda}^{(N)}$-symmetric and has $\mu_{\Lambda}^{(N)}$ as an invariant measure.

(ii) The diffusion process $\boldsymbol{M}_{\Lambda}^{(N)}$ is, up to $\mu_{\Lambda}^{(N)}$-equivalence, the unique diffusion process which has $\mu_{\Lambda}^{(N)}$ as symmetrizing measure and which solves the martingale problem for $\left(L_{\Lambda}^{(N)}, D\left(L_{\Lambda}^{(N)}\right)\right)$, i.e. for all $G \in D\left(L_{\Lambda}^{(N)}\right)$,

$$
G(\boldsymbol{X}(t))-G(\boldsymbol{X}(0))-\int_{0}^{t} L_{\Lambda}^{(N)} G(\boldsymbol{X}(s)) \mathrm{d} s, \quad t \geqslant 0,
$$

is an $\boldsymbol{F}_{\Lambda}^{(N)}(t)$-martingale under $\boldsymbol{P}_{\Lambda}^{(N)}(x)$ (hence starting in $x$ ) for $\mathcal{E}_{\Lambda}^{(N)}$-quasi all $x \in$ $\Gamma_{\Lambda}^{(N)}$.

In the above theorem $\boldsymbol{M}_{\Lambda}^{(N)}$ is canonical, i.e.

$$
\boldsymbol{\Omega}_{\Lambda}^{(N)}=C\left([0, \infty) \rightarrow \Gamma_{\Lambda}^{(N)}\right), \quad \boldsymbol{X}(t)(\omega)=\omega(t), \quad \omega \in \boldsymbol{\Omega}_{\Lambda}^{(N)} .
$$

The filtration $\left(\boldsymbol{F}_{\Lambda}^{(N)}(t)\right)_{t \geqslant 0}$ is the natural 'minimum completed admissible filtration' (see [13, Chapter A.2] or [20, Chapter IV]) obtained from the $\sigma$-algebras

$$
\sigma\left\{\omega(s) \mid 0 \leqslant s \leqslant t, \omega \in \Omega_{\Lambda}^{(N)}\right\}, \quad t \geqslant 0 .
$$

$\boldsymbol{F}_{\Lambda}^{(N)}:=\boldsymbol{F}_{\Lambda}^{(N)}(\infty):=\bigvee_{t \in[0, \infty)} \boldsymbol{F}_{\Lambda}^{(N)}(t)$ is the smallest $\sigma$-algebra containing all $\boldsymbol{F}_{\Lambda}^{(N)}(t)$ and $\left(\boldsymbol{\Theta}_{\Lambda}^{(N)}(t)\right)_{t \geqslant 0}$ are the corresponding natural time shifts. For a detailed discussions of these objects we refer the reader to $[\mathbf{2 0}]$. 
Proof. Due to Proposition 5.3, the proof is the same as that of Theorem 2.21.

Remark 5.5. Notice that the fact that $\operatorname{cap}_{\mathcal{E}_{\Lambda, N}}(D g)=0$, i.e. the $\mathcal{E}_{\Lambda, N}$-capacity of the set of diagonals in $\Lambda^{N}$ is zero, is essential for proving Proposition 5.3, which in turn yields Theorem 5.4. In situations $(\mathrm{A})-(\mathrm{C})$ this is due to the fact that the interaction potential $\phi$ is repulsive (see conditions (RP) or (SRP)). Thus, from conditions (RP) or (SRP) we obtain that the $N$-particle stochastic dynamics $\boldsymbol{M}_{\Lambda}^{(N)}$ in $\Lambda$ stays in the configuration space $\Gamma_{\Lambda}^{(N)}$ of single configurations, i.e. we never have more then one particle in one position. In other words, the repulsive interaction potential $\phi$ prevents particles from interpenetrating each another. In situation (D) we obtain $\operatorname{cap}_{\mathcal{E}_{\Lambda, N}}(D g)=0$ by the fact that the diagonals have codimension at least 2 . This, of course, is only true for $d \geqslant 2$.

Another way to construct the process $\boldsymbol{M}_{\Lambda}^{(N)}$ is to use first Corollary 4.8 to construct the corresponding process in $\left\{\varrho_{\Lambda, N}>0\right\}=\Lambda^{N} \backslash D g=\tilde{\Lambda}^{N}$ and then the mapping $\operatorname{sym}^{(N)}: \tilde{\Lambda}^{N} \rightarrow \Gamma_{\Lambda}^{(N)}$ to construct $\boldsymbol{M}_{\Lambda}^{(N)}$ as the image process under sym ${ }^{(N)}$. This approach also needs $\operatorname{cap}_{\mathcal{E}_{\Lambda, N}}(D g)=0$, which in this situation yields that the process in $\Lambda^{N}$ does not hit the diagonals $D g \subset \Lambda^{N}$. This is of essential importance, since otherwise it would not be possible to apply the mapping $\operatorname{sym}^{(N)}$, which is only defined outside the diagonals.

Acknowledgements. We thank Yuri Kondratiev, Michael Röckner and Karl-Theodor Sturm for discussions and helpful comments. Financial support through the Schwerpunkt Mathematik und Praxis, University of Kaiserslautern, is gratefully acknowledged.

\section{References}

1. S. Albeverio AND M. RöCKNER, Dirichlet form methods for uniqueness of martingale problems and applications, in Stochastic Analysis, Ithaca, NY, 1993, Proceedings of Symposia in Pure Mathematics, Volume 57, pp. 513-528 (American Mathematical Society, Providence, RI, 1995).

2. S. Albeverio, Yu. G. Kondratiev and M. Röckner, Strong Feller properties for disorted Brownian motion and applications to finite particle systems with singular interactions, in Finite and infinite dimensional analysis, in honor of Leonard Gross, Contemporary Mathematics, Volume 317 (American Mathematical Society, Providence, RI, 2003).

3. S. Albeverio, W. Schachermayer and M. TAlagrand, Lectures on probability theory and statistics, Lecture Notes in Mathematics, Volume 1816 (Springer, 2003).

4. H. W. Alt, Lineare Funktionalanalysis (Springer, 2002).

5. R. F. BASS AND P. Hsu, The semimartingale structure of reflecting Brownian motion, Proc. Am. Math. Soc. 108 (1990), 1007-1010.

6. R. F. BASS AND P. Hsu, Some potential theory for reflecting Brownian motion in Hölder and Lipschitz domains, Annals Probab. 19 (1991), 486-508.

7. Z.-Q. Chen, On reflecting diffusion processes and Skorokhod decompositions, Prob. Theory Relat. Fields 94 (1993), 281-315.

8. T. Fattler and M. Grothaus, Strong Feller properties for distorted Brownian motion with reflecting boundary condition and an application to continuous $N$-particle systems with singular interactions, J. Funct. Analysis 246 (2007), 217-241.

9. M. Fradon, Diffusions réfléchies réversibles dégénérées, Potent. Analysis 6 (1997), 369414. 
10. M. Fukushima, Dirichlet forms and Markov processes (North-Holland, Amsterdam, 1980).

11. M. Fukushima, Energy forms and diffusion processes, in Mathematics + physics: lectures on recent results (ed. L. Streit), Volume 1, pp. viii, 338 (World Scientific, Singapore, 1985).

12. M. Fukushima And M. TOMisaki, Construction and decomposition of reflecting diffusions on Lipschitz domains with Hölder cusps, Prob. Theory Relat. Fields 106 (1996), $521-557$.

13. M. Fukushima, Y. Oshima And M. TAKeda, Dirichlet forms and symmetric Markov processes (de Gruyter, New York, 1994).

14. D. Gilbarg And N. S. Trudinger, Elliptic partial differential equations of second order (Springer, 1977).

15. M. Grothaus, Spectral gap of the continuous $N$-particle stochatic dynamics with singular interactions in a finite volume, in preparation (2008).

16. M. Grothaus, Yu. G. Kondratiev and M. Röckner, $N / V$-limit for stochastic dynamics in continuous particle systems, Prob. Theory Relat. Fields 137 (2007), 121160 .

17. P.-L. Lions And A.-S. Sznitman, Stochastic differential equations with reflecting boundary conditions, Commun. Pure Appl. Math. 37 (1984), 511-537.

18. T. J. LyOns AND T. S. ZhANG, Decomposition of Dirichlet processes and its applications, Annals Probab. 22 (1994), 494-524.

19. T. J. LyOns AND W. A. Zheng, A crossing estimate for the canonical process on a Dirichlet space and a tightness result, Astérisque 157 (1988), 249-271.

20. Z.-M. MA AND M. RÖCKNER, Introduction to the theory of (non-symmetric) Dirichlet forms (Springer, 1992).

21. E. Pardoux And R. J. Williams, Symmetric reflected diffusions, Annales Inst. H. Poincaré 30 (1994), 13-64.

22. K.-T. STURM, Sharp estimates for capacities and applications to symmetric diffusions, Prob. Theory Relat. Fields 103 (1995), 73-89.

23. G. Trutnau, Skorokhod decomposition of reflected diffusions on bounded Lipschitz domains with singular non-reflection part, Prob. Theory Relat. Fields 127 (2003), 455495.

24. R. J. Williams and W. A. Zheng, On reflecting Brownian motion: a weak convergence approach, Annales Inst. H. Poincaré B 26 (1990), 461-488. 